\title{
Chow-Liu Trees Are Sufficient Predictive Models For Reproducing Key Features of Functional Networks of Periictal EEG Time-Series
}

\author{
Andreas Steimer ${ }^{\dagger *}$, Frédéric Zubler ${ }^{\dagger}$ and Kaspar Schindler ${ }^{\dagger}$ \\ $\dagger$ Department of Neurology, Inselspital $\backslash$ Bern University Hospital $\backslash$ University Bern, \\ Bern, Switzerland
}

\begin{abstract}
Seizure freedom in patients suffering from pharmacoresistant epilepsies is still not achieved in $20-30 \%$ of all cases. Hence, current therapies need to be improved, based on a more complete understanding of ictogenesis. In this respect, the analysis of functional networks derived from intracranial electroencephalographic (iEEG) data has recently become a standard tool. Functional networks however are purely descriptive models and thus are conceptually unable to predict fundamental features of iEEG time-series, e.g. in the context of therapeutical brain stimulation.

In this paper we present some first steps towards overcoming the limitations of functional network analysis, by showing that its results are implied by a simple predictive model of time-sliced iEEG time-series. More specifically, we learn distinct Graphical models (so called Chow-Liu(CL) trees) as models for the spatial dependencies between iEEG signals. Bayesian inference is then applied to the CL trees, allowing for an analytic derivation/prediction of functional networks, based on thresholding of the absolute value Pearson correlation coefficient $(\mathrm{CC})$ matrix. Using various measures, the thus obtained networks are then compared to those which were derived in the classical way from the empirical CC-matrix. In the high threshold limit we find (a) an excellent agreement between the two networks and (b) key features of periictal networks as they have previously been reported in the literature.

Apart from functional networks, both matrices are also compared element-wise, showing that the CL approach leads to a sparse representation, by setting small correlations to values close to zero while preserving the larger ones.

Overall, this paper shows the validity of CL-trees as simple, spatially predictive models for periictal iEEG data. Moreover, we suggest straightforward generalizations of the CL-approach for modeling also the temporal features of iEEG signals.
\end{abstract}

Keywords: Epilepsy, periictal EEG, predictive modeling, Bayesian inference, graphical models, Chow-Liu tree, Chow-Liu algorithm, functional networks, minimum spanning tree

Abbreviations: CL, Chow-Liu; CC, (Pearson) correlation coefficient; iEEG, intracranial EEG; GM, graphical model; SOZ, seizure onset zone; HMM, Hidden Markov Model

${ }^{*}$ Corresponding author: Department of Neurology $\backslash$ Inselspital, Freiburgstrasse 4, 3010-Bern $\backslash$ Switzerland, Tel.: +41 (0)31 63245 47, Email: Andreas.Steimer@insel.ch 


\section{Introduction}

Epilepsy is the most prevalent chronic neurologic disorder and affects 50 million people worldwide (Meyer et al., 2010). In 2005, epilepsy was defined as a disorder of the brain, characterized by an enduring predisposition to generate epileptic seizures (Fisher et al., 2005). Seizures may cause transient changes of behavior and perception and may lead to accidents or fatal systemic dysregulations like heart arrhythmia (Bozorgi \& Lhatoo, 2013) or hypoxia. In addition, the unpredictability of seizure occurrences (Mormann et al., 2007) and the fear of having a seizure in public are often the reasons for social withdrawal and reactive depression. For all these reasons, the primary goal of epilepsy treatment is to achieve permanent seizure freedom. However, despite many new drugs and modern resective and neuromodulatory surgical techniques, seizure freedom is still not achieved in approximately 20-30\% of the patients (Cascino, 2008). Therefore it is mandatory to improve current therapies.

A recent approach to guide further therapeutic developments is to better understand the functional brain networks and their dynamics, that are associated with epileptic seizures (Engel et al., 2013; Richardson, 2012). Functional brain networks are mathematical graph objects consisting of nodes and links, which connect pairs of nodes (Bullmore \& Sporns, 2009; Rubinov \& Sporns, 2010). In EEG-derived functional networks, each node of the network stands for a single EEG signal and a link between two nodes represents a measure of interdependency between the corresponding pair of EEG signals. The goal of functional network analysis in epilepsy research is to identify the critical parts of the brain, that is, the "hubs" of these networks, that have the strongest influence on the dynamics of the network as a whole (Stam \& van Straaten, 2012; Zubler et al., 2014). It is expected that targeting only the hubs of functional networks would make therapies more effective and potentially even less invasive. In the past, there have been a number of studies aiming at 1) the definition of different types of functional networks -based on various EEG-derived signal interdependencies used to define the networks links (edges), and 2) the investigation of how distinct topological properties of these networks change before, during and after epileptic seizures (Ponten et al., 2007; Schindler et al., 2008; Kramer et al., 2010; Wilke et al., 2011; van Mierlo et al., 2011; Varotto et al., 2012). For example, in (Schindler et al., 2008; Kramer et al., 2010) it was found that initial ictal network dynamics are characterized by strong modularization, i.e. a fragmentation of the network into a set of strongly intra- but weakly interconnected subnetworks. In terms of the underlying EEG signals, this indicates a dynamical shift during early seizure development towards locally highly synchronized 'islands' of EEG activity, that are desynchronized amongst each other. These and related findings (Schindler et al., 2007) have lead to a paradigm shift in epilepsy research, which had previously been dominated by the idea of seizures being events of temporally constant, global hypersynchrony (Schindler et al., 2007; Jiruska et al., 2013).

Despite these achievements, a fundamental shortcoming of the functional network approach is its purely descriptive nature, and consequently, its inability to generate predictions regarding the spatio-temporal dynamics of periictal iEEG time-series. For example, functional networks are conceptually unable to draw any inferences about the probability of states of some subset of the iEEG electrodes, given that the remaining ones are held fixed to 
specific states, e.g. by means of artificial brain stimulation. Predictive models like graphical models(GMs) in contrast could have an important impact on epilepsy treatment, ranging from inferences of the seizure onset zone, the area of the brain where ictal iEEG activity starts, to the testing of brain stimulation protocols that aim at suppression of such activity.

In this paper we present some first steps towards overcoming the limitations of functional network analysis, by showing that its results are implied by a simple predictive model of time-sliced iEEG time-series. More specifically, we learned distinct GMs (so called Chow$\mathrm{Liu}(\mathrm{CL})$ trees) from a seizure database consisting of 93 seizures and compared the results of Bayesian inference in these CL-trees to the results obtained from analyzing the raw, periictal data directly. Comparisons were done based on (a) direct comparisons between the Pearson correlation coefficient matrices derived from the CL-trees and from the raw data respectively; and (b) the functional networks that were constructed from these two types of matrices. With respect to several measures of functional network connectivity, we found an excellent agreement between both types of networks, indicating that the results of functional network analysis are implied by CL-trees. Furthermore, the learned CL-trees yielded "sparse" correlation matrices, which accurately matched the strong correlations and set the weaker ones to values close to zero. While our results do not imply the applicability of CL-trees for modeling all detailed aspects of periictal time-series in general, they still show their validity as predictive models for the functional network connectivity of such data.

\section{Materials \& Methods}

\subsection{Patient and Periictal iEEG Data}

We included periictal, intracranial EEG (iEEG) time-series of variable length recorded from 25 consecutive patients ( 15 females) in our study (ref table 1 ). The patients suffered from pharmacoresistant epilepsies and underwent long-term iEEG monitoring at the department of Neurology of the University Hospital in Bern. The mean age ( \pm 1 standard deviation) was $30 \pm 12 y$ (range 7-58y). Except for the need for invasive EEG studies, there were no additional inclusion criteria. Specifically, patients with and without radiologically detectable lesions were enrolled. Electrodes implanted consisted of intracranial strip-, depth- and grid electrodes all manufactured by AD-TECH (Racine, WI, U.S.A). Distance of neighboring electrode contacts was $1 \mathrm{~cm}$ for strip- and grid electrodes and $0.5 \mathrm{~cm}$ or $1 \mathrm{~cm}$ for depth electrodes. For typical locations and numbers of implanted electrode contacts see columns "SOZ" and "El.Sz." respectively in table 1. Mean number of implanted electrode contacts ( \pm 1 standard deviation) was $60.3 \pm 15.5$. Each of the iEEG time-series recorded from the contacts covered a complete seizure, plus the preceding preictal and the subsequent postictal epochs, each lasting for 180s. At most 5 seizures per patient, each lasting for at least 30s and for at most 500s, were included, making up 93 seizures in total. Retrospective data analysis had been approved by the Ethics Committee of the Kanton of Bern. In addition, all patients gave written and informed consent that their data from long-term video-EEG recordings might be used for research or teaching purposes. 


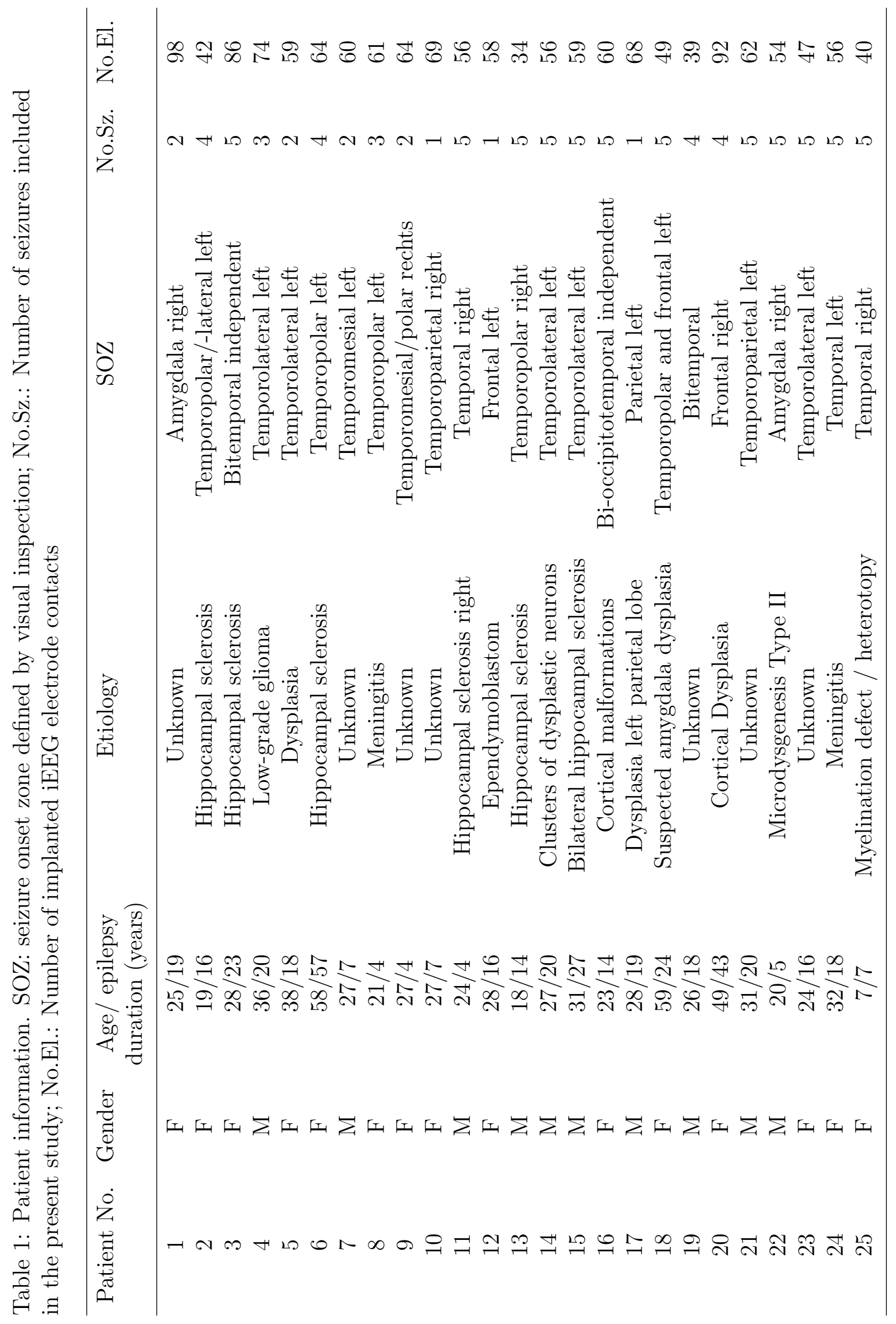




\subsection{Signal Filtering, Amplitude And Time Discretization Of The Empirical iEEG Data}

To stay as close as possible to the protocol used in the comparison literature (Kramer et al., 2010), our analysis is based on broadband iEEG signals, sampled at 512Hz. Filtering consisted, first, of anti-aliasing within the C64 amplifier of the NicoletOne recording system (VIASYS Healthcare, Inc., Madison, WI, U.S.A.) that was used. Signal attenuation was $-16 \mathrm{~dB}$ at $512 \mathrm{~Hz}$. Subsequent forward/backward bandpass filtering based on a fourth-order butterworth filter constrained the signal further to the $[0.5150] \mathrm{Hz}$ frequency range. We refer to the thus obtained signal as the raw iEEG time-series or data.

Because the Chow-Liu algorithm is defined for discrete data (Chow \& Liu, 1968; Meila \& Jordan, 2001), we used the following scheme to discretize (symbolize) the iEEG data:

First, for each iEEG channel, the raw signal was normalized, i.e. centered to mean zero and scaled to standard-deviation $\sigma=1$. We then discretized the signal by quantizing its amplitude using a grid of $m=6$ equidistant bins within the interval $[-k \sigma, k \sigma], k=1$. Values outside this interval were clamped to the closest of its boundaries $\pm k \sigma$.

The values chosen for $m$ and $k$ are a compromise between the faithful preservation of the original, quasi-analog iEEG signal and computational requirements (avoidance of excessive processing time and overfitting by the Chow-Liu algorithm). The latter point is of particular importance; since the algorithm is based on estimating all joint histograms of variable pairs (see section 2.3.2), those histograms may be severely corrupted by sampling noise, if the squared number $m^{2}$ of joint bins is too large compared to the number of data points within a time-window. In this case there is not enough data available to accurately represent the true pairwise joint probabilities and these errors then lead to a falsely factorized representation of the full joint probability (see eq. 2.1). So, restricting the number of bins per variable is not a matter of computational cost alone, but also of representational accuracy.

Regarding parameter $k$, the $\pm k \sigma$ limits were not of concern for pre- and postictal time points, since the standard deviation of each iEEG signal was dominated by the large amplitude oscillations that occur during seizure progression (see fig.2). Hence, pre- and postictally the $\pm k \sigma$ limits were barely reached. On the other hand, ictal signals may be significantly larger and often cross these limits. However, because ictal activity is dominated by oscillations at some characteristic frequency, it is only the stereotyped upper/lower part of an oscillatory wave that is "chopped off" through amplitude discretization. For ictal signals, such a procedure can therefore be effectively approximated by a multiplicative scaling of the amplitude that leaves phase information unchanged. Hence, it is the unperturbed phase shifts that determine the correlation structure of a set of quasi-sinusoidal signals, because the Pearson correlation coefficient is insensitive to multiplicative scalings.

For these reasons, the results in this paper are robust against at least small perturbations of $m$ and $k$ (e.g. for the setting $m=8, k=1.5$, not shown). As we will show in fig. 3, the used settings $m=6, k=1$ are sufficient for reproducing the Pearson correlation coefficient matrices of the quasi-analog signals to a good degree. Moreover, as we will discuss in section 3.2 , fig. 8 , there is virtually no influence of those parameters on the properties of functional networks derived from Pearson correlation coefficient matrices. 
For a given seizure, the discretized iEEG time-series resulting from the symbolization procedure were partitioned into overlapping windows of $2.5 \mathrm{~s}$ length. The temporal difference between the times of onset of two consecutive windows was set to $2 \mathrm{~s}$, creating an overlap of $20 \%$. The same set of windows was used for partitioning each iEEG channel of a given seizure. For each partition, a Chow-Liu tree was learned separately, from which the absolute value Pearson correlation coefficient matrix was computed by Bayesian inference (see following sections).

\subsection{Modeling Of The Preprocessed iEEG Data}

\subsubsection{The Chow-Liu Tree As A Simple Predictive Model}

A Chow-Liu (CL) tree (Chow \& Liu, 1968; Meila \& Jordan, 2001; Murphy, 2012) is a specific, simple type of Graphical Model (GM) (Löliger, 2004; Bishop, 2006; Koller \& Friedman, 2009; Murphy, 2012) for a given set (vector) of variables $\mathbf{x}=\left(x_{1}, \ldots, x_{n}\right)$, which in our case corresponds to a set of iEEG signals. In general, a GM is a graph that represents the (in)dependencies between the variables in $\mathbf{x}$ and hence is defined by a set of nodes $\mathcal{V}=(1, \ldots, n)$-which correspond to the individual variables $x_{i}$ - and a set of edges $\mathcal{E} \subseteq \mathcal{V}^{2}$ connecting pairs of these nodes. Each edge set $\mathcal{E}$ implies a distinct pattern of (in)dependencies between the variables and in case of CL-trees $\mathcal{E}$ is constrained to spanning trees, i.e. graphs that entail a single, distinct path between each pair of nodes. Moreover, for a spanning tree to be a CL-tree $\mathcal{E}$ has to meet a certain optimality condition that we will discuss in the next section. While displaying dependencies between variables in a graphical way, a GM is also a factorized representation of some joint probability distribution $P(\mathbf{x})$, which respects the (in)dependencies implied by the GMs edge set. In case of trees, this distribution is given by (Meila \& Jordan, 2001):

$$
P(\mathbf{x})=\frac{\prod_{(i, j) \in \mathcal{E}} P_{i j}\left(x_{i}, x_{j}\right)}{\prod_{i \in \mathcal{V}} P_{i}\left(x_{i}\right)^{\operatorname{deg}(i)-1}}
$$

where $\operatorname{deg}(i)$ is the branching degree, i.e. the number of neighboring nodes of node $i$. Equation 2.1 decomposes $P(\mathbf{x})$ into the product of those pairwise joint probabilities $P_{i j}\left(x_{i}, x_{j}\right)$, which correspond to the edge set $\mathcal{E}$ and divides the result by the product of the marginal probabilities $P_{i}\left(x_{i}\right)$ of the individual variables. The GM defined by $P(\mathbf{x})$ can be used either as a generative model (by sampling artificial data points from $P(\mathbf{x})$ ), or as a predictive model (by inferring conditional probability distributions $P\left(\mathbf{x}_{u} \mid \mathbf{x}_{o}\right)$ for arbitrary subsets $\left.O, U \subset\left\{x_{1}, \ldots, x_{n}\right\}\right)$. In the latter case, for example, one may predict the joint distribution of voltages $\mathbf{x}_{u}$ of some subset of unobserved electrodes $U$, given that electrodes of another (observed) subset $O$ are held fixed to specific voltage levels, described by vector $\mathbf{x}_{o}$. Hence, on the basis of probability distributions, GMs allow for predictions under prespecified environmental scenarios.

In this paper, we follow the route of such predictive modeling, by inferring all $\frac{1}{2} n(n-1)$ possible joint distributions of variable pairs, for the definition of functional networks from 
the resulting Pearson correlation coefficient matrix (see sections 2.4.1 and 2.5.1). Hereby the set of observed variables is the empty set $O=\emptyset$. Moreover, the single variable and pairwise marginal probabilities in eq.2.1 may be used as a starting point for computing the Shannon entropy $H$ of $P(\mathbf{x})$. As we will discuss later, for CL-trees the dynamics of $H(\mathbf{x})$ are consistent with previous results from the analysis of periictal iEEG data. Based on eq.2.1 $H(\mathbf{x})$ can be computed in the following way:

$$
\begin{aligned}
H(\mathbf{x}): & =-\sum_{\mathbf{x}} P(\mathbf{x}) \cdot \log P(\mathbf{x}) \\
& =-\sum_{(i, j) \in \mathcal{E}} \sum_{x_{i}, x_{j}} P_{i j}\left(x_{i}, x_{j}\right) \cdot \log P_{i j}\left(x_{i}, x_{j}\right)+\sum_{i \in \mathcal{V}}(\operatorname{deg}(i)-1) \sum_{x_{i}} P_{i}\left(x_{i}\right) \cdot \log P_{i}\left(x_{i}\right) \\
& =\sum_{(i, j) \in \mathcal{E}} H\left(x_{i}, x_{j}\right)-\sum_{i \in \mathcal{V}}(\operatorname{deg}(i)-1) H\left(x_{i}\right)
\end{aligned}
$$

\subsubsection{The Chow-Liu Algorithm For Finding The Chow-Liu Tree}

The goal of the CL algorithm is to find the CL-tree, i.e. an optimal spanning tree, from a given data set for all variables $\mathbf{x}$ (in our case, this data set is given by the windowed and discretized iEEG time-series, see section 2.2). More specifically, the CL algorithm aims at minimizing the Kullback-Leibler divergence $D\left(P^{*} \| P\right)$ between the empirical distribution $P^{*}(\mathbf{x})$ (i.e. the true distribution underlying the empirical data set) and a model distribution $P(\mathbf{x})$, restricted to a tree distribution (ref. equation 2.1). Minimization is performed along both, the set of parameters $P_{i j}\left(x_{i}, x_{j}\right),(i, j) \in \mathcal{E}$ defining the tree distribution for a given edge set $\mathcal{E}$ and the edge set itself. Minimizing w.r.t. ${ }^{1}$ the parameters yields the condition

$$
P_{i j}\left(x_{i}, x_{j}\right)=P_{i j}^{*}\left(x_{i}, x_{j}\right), \forall(i, j) \in \mathcal{E}
$$

That is, the pairwise joint probabilities of the tree model have to be set equal to the corresponding empirical probabilities $P_{i j}^{*}\left(x_{i}, x_{j}\right)$, which can be obtained directly by computing pairwise histograms of the data. When $P(\mathbf{x})$ in eq. 2.1 is inserted into the defining expression of the KL-divergence $D\left(P^{*} \| P\right)$ and provided condition 2.5 is fullfilled, one obtains the following sum of mutual-information and entropy values as an expression for $D\left(P^{*} \| P\right)$ (Chow \& Liu, 1968):

$$
D\left(P^{*} \| P\right)=-\sum_{(i, j) \in \mathcal{E}} I^{*}\left(x_{i} ; x_{j}\right)+\sum_{i \in \mathcal{V}} H^{*}\left(x_{i}\right)-H^{*}(\mathbf{x})
$$

where $I^{*}(\cdot)$ and $H^{*}(\cdot)$ refer respectively to the mutual-information and entropy of the empirical distribution. Since the terms $\sum_{i \in \mathcal{V}} H^{*}\left(x_{i}\right)$ and $H^{*}(\mathbf{x})$ are independent of tree structure, it follows directly from eq. 2.6 that $D\left(P^{*} \| P\right)$ becomes minimal w.r.t. to tree structure, if $\mathcal{E}$ is chosen such that $-\sum_{(i, j) \in \mathcal{E}} I\left(x_{i} ; x_{j}\right)$ is minimal (while maintaining the spanning tree

\footnotetext{
${ }^{1}$ with respect to
} 
requirement). Therefore if, for an edge connecting nodes $i$ and $j$, the values $-I^{*}\left(x_{i} ; x_{j}\right)$ are interpreted as edge weights, minimizing eq. 2.6 w.r.t. $\mathcal{E}$ becomes a minimum spanning tree problem, for which efficient solution methods exists, most notably Kruskal's algorithm (Kruskal, 1956) and Prim's algorithm (Prim, 1957).

Either solution method may be employed by the CL algorithm, for the technical implementation of which we have made use of the 'Probabilistic Modeling Toolkit(PMTK3)' (publicly available under https://github.com/probml/pmtk3). The CL algorithm in PMTK3 is based on Prim's algorithm.

\subsection{Construction, Analysis And Comparison Of (Absolute Value) Pearson Correlation Coefficient Matrices}

\subsubsection{Derivation Of (Absolute Value) Pearson Correlation Coefficient Matrices From The Empirical Data And The Model}

Pearson correlation coefficient matrices were derived from the raw, continuous iEEG data, discretized iEEG data and the distributions

$P_{i j}\left(x_{i}, x_{j}\right),(i, j) \in \mathcal{V}^{2}$ inferred from the CL-trees. Throughout this paper we will denote by $\mathbf{s C C}=\left(s c c_{i j}\right)$ the ordinary (signed) Pearson correlation-coefficient matrix (but with zero main diagonal) and with $\mathbf{C C}=\left(\left|s c c_{i j}\right|\right)$ the corresponding absolute value Pearson correlation-coefficient matrix, from which functional networks were constructed. In this context, the superscripts $\mathbf{R}, \mathbf{D}, \mathbf{M}$ of some matrix refer respectively to the matrix constructed from the raw data, discretized data and the CL-tree model. In case of the raw data, the bandpass filtered iEEG time-series of a given seizure were partitioned, based on the same set of windows that was used for partitioning the discretized time-series (section 2.2). For each such partition, the matrices $\mathbf{s} \mathbf{C C}^{\mathbf{R}}$ and $\mathbf{C C}^{\mathbf{R}}$ were computed directly from the raw data.

The exact same procedure was applied to the partitioned, discretized data, yielding matrices $\mathbf{s} \mathbf{C C}^{\mathbf{D}}$ and $\mathbf{C C}^{\mathbf{D}}$.

In case of the CL trees, the partitioned, discretized data served as starting point for the construction of CL-trees, by learning an individual tree model for each partition (see

section 2.3.2). Using results obtained from probabilistic inference, the matrices $\mathbf{s} \mathbf{C C}^{\mathbf{M}}$ and $\mathbf{C C}^{\mathbf{M}}$ of each CL-tree were analytically computed based on the mathematical definitions of 
covariance, variance and Pearson correlation:

$$
\begin{aligned}
s c c_{i j}^{M} & :=\left(1-\delta_{i j}\right) \frac{\operatorname{Cov}\left[x_{i}, x_{j}\right]}{\sqrt{\operatorname{Var}\left[x_{i}\right] \operatorname{Var}\left[x_{j}\right]}} \\
c c_{i j}^{M} & :=\left|s c c_{i j}^{M}\right| \\
\operatorname{Cov}\left[x_{i}, x_{j}\right] & :=\mathbb{E}\left[x_{i} \cdot x_{j}\right]-\mathbb{E}\left[x_{i}\right] \cdot \mathbb{E}\left[x_{j}\right] \\
& =\left(\sum_{x_{i}=1, x_{j}=1}^{m} x_{i} \cdot x_{j} \cdot P_{i j}\left(x_{i}, x_{j}\right)\right)-\left(\sum_{x_{i}=1}^{m} x_{i} \cdot P_{i}\left(x_{i}\right)\right) \cdot\left(\sum_{x_{j}}^{m} x_{j} \cdot P_{j}\left(x_{j}\right)\right) \\
\operatorname{Var}\left[x_{i}\right] & :=\mathbb{E}\left[x_{i}^{2}\right]-\mathbb{E}^{2}\left[x_{i}\right]=\left(\sum_{x_{i}=1}^{m} x_{i}^{2} \cdot P_{i}\left(x_{i}\right)\right)-\left(\sum_{x_{i}=1}^{m} x_{i} \cdot P_{i}\left(x_{i}\right)\right)^{2} \\
P_{i}\left(x_{i}\right) & =\sum_{x_{j}=1}^{m} P_{i j}\left(x_{i}, x_{j}\right)
\end{aligned}
$$

Hence, for computing these quantities only the pairwise, joint probabilities

$P_{i j}\left(x_{i}, x_{j}\right), \forall(i, j) \in \mathcal{V}^{2}$ needed to be inferred from the CL-trees. If $(i, j) \in \mathcal{E}$, then the corresponding probabilities can be obtained directly from the results of the CL algorithm, i.e. from the histograms of the discretized, empirical data (section 2.3.2). In all other cases however, true probabilistic inference must be applied to the CL-trees, by marginalizing (summing) out all variables $x_{k}, k \in \mathcal{V} \backslash\{i, j\}$ in eq. 2.1. For that, PMTK3 makes use of the Junction-Tree algorithm (Aji \& McEliece, 2000), which is guaranteed to provide exact results.

\subsubsection{Elementwise Comparison Of Pearson Correlation Coefficient Matrices}

We compared the elements of matrices $\mathbf{s} \mathbf{C C}^{\mathbf{M}}$ to those of their $\mathbf{s} \mathbf{C C}^{\mathbf{R}}$ counterparts in the following way:

First, each seizures sequence of $\mathbf{s} \mathbf{C C}^{\mathbf{R}}$ and $\mathbf{s} \mathbf{C C}^{\mathbf{M}}$ matrices (with one pair of matrices for each partitioning window) was temporally divided into a set of 12 epochs, ranging from $\operatorname{prI}$ (preictal epoch) over $I_{1}, \ldots, I_{10}$ (equidistant ictal epochs) to $p o I$ (postictal epoch) (Schindler et al., 2008; Kramer et al., 2010). Subsequently, for any given matrix $\mathbf{s C C}^{\mathbf{M}}$ from any epoch, we sorted all of its elements $s c c_{i j}^{M}$ into 19 equidistant bins, whose centers ranged from $\{-0.94, \ldots, 0, \ldots, 0.94\}$. For each such bin $B_{k}, k \in\{-0.94, \ldots, 0, \ldots, 0.94\}$ we then formed the level set $s C C_{k}^{R}:=\left\{s c c_{i j}^{R} \mid(i, j) \in \mathcal{V}^{2}, s c c_{i j}^{M} \in B_{k}\right\}$ of elements of the $\mathbf{s} \mathbf{C C}^{\mathbf{R}}$-matrix that corresponds to the same partitioning window. In other words, we formed potentially overlapping bins along the $s c c_{i j}^{R}$-axis, each of which is associated with a bin $B_{k}$ along the $s c c_{i j}^{M}$ axis. Subsequently, we took the mean $\left\langle s C C_{k}^{R}\right\rangle$ and standard deviation $\sigma\left(s C C_{k}^{R}\right)$ across the level set, which respectively served as measures of matching accuracy and sparsity. Ideally, for large average matching accuracy between matrix elements $s C C_{i j}^{R}$ and $s C C_{i j}^{M}$, the mean $\left\langle s C C_{k}^{R}\right\rangle$ should be unbiased and consequently be close to $k$, whereas a high sparsity degree of $\mathbf{s C C}^{\mathbf{M}}$ is given by a data spread $\sigma\left(s C C_{k}^{R}\right)$ that is fairly large for $k=0$ and falls of 
steeply for $|k|>0$. In this case, provided high average matching accuracy is also given, small correlations $s C C_{i j}^{R}$ are set to values close to zero by the CL-model, whereas large correlations are faithfully preserved, indicating a high degree of sparsity of matrix $\mathbf{s C C} \mathbf{C}^{\mathbf{M}}$.

For a given seizure, we averaged both measures across all level sets, whose corresponding partitioning windows belonged to the same seizure epoch (i.e. prI, $\mathrm{I}_{1}, \ldots, \mathrm{I}_{10}, \mathrm{poI}$ ). Separated by epoch, these mean values were averaged again across all seizures of the database, to produce the results of figure 5 .

\subsubsection{Construction Of Surrogate Time-Series / Analysis Of P-Values Of Pear- son Correlation Coefficient Matrices}

Because correlation coefficients of the $\mathbf{C C}^{\mathbf{R}}$ matrices were determined from a finite set of iEEG data, the observed values could possibly be explained by random correlations of in fact uncorrelated iEEG time-series. To determine the likelihood of such an event, we have computed p-values from artificial, uncorrelated, surrogate time-series, that preserve the amplitude distribution and autocorrelation function of the original, unprocessed (i.e. not discretized) iEEG signals (Schreiber \& Schmitz, 2000). More specifically, for each matrix $\mathbf{s C C}^{\mathbf{R}}$, we have also computed an associated matrix $\mathbf{P}$, whose elements $p_{i j}$ gave the p-value of the corresponding element $s c c_{i j}^{R}$. For signals $i$ and $j$, p-values $p_{i j}$ were computed from the most extreme end of the empirical distributions (histograms) of correlation coefficients. Therefore, the maximally possible p-value was $p_{i j}=0.5$. Histograms of correlation coefficients were extracted from 100 pairs of surrogate time-series.

Based on a procedure similar to the one described in section 2.4.2, p-values resulting from the whole seizure database were used to verify if the CL-model generally eliminates (i.e. sets to values close to zero) those correlations $s c c_{i j}^{M}$ for which randomness cannot be excluded. More specifically, for each bin $B_{k}, k \in\{-0.94, \ldots, 0, \ldots, 0.94\}$ along the $s c c_{i j}^{M}$-axis we examined the empirical distribution of elements from the set $P_{k}:=\left\{p_{i j} \mid(i, j) \in \mathcal{V}^{2}, s c c_{i j}^{M} \in B_{k}\right\}$. Ideally, if a large $\left|s c c_{i j}^{M}\right|$ indeed signals nonrandomness of its partner $s c c_{i j}^{R}$, we expect the corresponding $p_{i j}$ to be small. Thus, for $|k| \rightarrow 1$ the elements of $P_{k}$ are expected to become tightly distributed around zero (indicated by a mean $\left\langle P_{k}\right\rangle \approx 0$ and standard deviation $\left.\sigma\left(P_{k}\right) \approx 0\right)$. Conversely, if a small $\left|s c c_{i j}^{M}\right|$ signifies that the null hypothesis of a purely random $\left|s c c_{i j}^{R}\right|$ counterpart cannot be excluded, then for $|k| \rightarrow 0$ the p-values in $P_{k}$ should become redistributed towards larger values. In fact, for any null hypothesis that is known to be true, the p-values obtained from any valid statistical testing method follow a uniform distribution (Bland, 2013), which in our case is a uniform distribution on [0,0.5]. Therefore, a failure to match the distribution of p-values in $P_{k=0}$ with a uniform distribution serves to refute the null hypothesis of small $\left|s c c_{i j}^{M}\right|$ being associated with random correlations $\left|s c c_{i j}^{R}\right|$. Thus, we performed a hypothesis test based on the following null hypothesis

$$
\begin{aligned}
& H_{0} \text { : for } k=0 \text { elements of the level set } s C C_{k}^{R} \text { are due to type I errors, } \\
& \text { i.e. they are random correlations from in fact independent time series }
\end{aligned}
$$

For refuting $H_{0}$ it is therefore sufficient to show that the mean $\left\langle P_{k=0}\right\rangle$ is significantly different from 0.25 , the mean of a uniform distribution on the interval $[00.5]$. Hence, we first computed 
the mean across the union of all those $P_{k=0}$ that belong to the same seizure and epoch and subsequently performed one-sample t-tests across all seizures, that is, one separate test for each epoch. This way, application of the t-test was valid, since each tested epoch-restricted data set contained exactly 93 independent mean p-values, that is, one mean value for each seizure. Testing was done against the hypothesized mean of 0.25 .

\subsection{Construction, Analysis And Comparison Of Functional Net- works Derived From The Absolute Value Pearson Correlation Coefficient Matrices}

\subsubsection{Construction of Functional Networks from the Model and the Empirical Data}

Functional networks were constructed based on both, the CC-matrices computed from the raw data and the CC-matrices inferred from the CL-trees. In the latter case, it is important to keep in mind, that there are two qualitatively different networks at play; The CL-tree is a generative and predictive graphical model, which allows for the generation of artificial data and the prediction of marginal distributions. From these marginal distributions it is then possible to derive a CC-matrix that can be used as a starting point for the construction of functional networks, which are neither generative nor predictive models.

Weighted, undirected functional networks (Rubinov \& Sporns, 2010) were constructed based on edge sets that were defined by thresholding the elements of $\mathbf{C C}^{\mathbf{M}}$ matrices. Elements $c c_{i j}^{M}$ above the threshold were kept unaffected, whereas those below the threshold were set to zero. The thus defined thresholding 'mask' for $\mathbf{C C}^{\mathbf{M}}$ was then applied also to the $\mathbf{C C}^{\mathbf{R}}$ matrix that corresponded to the same iEEG data partition (window) as matrix $\mathbf{C C}^{\mathbf{M}}$. In other words, networks defined by $\mathbf{C C}^{\mathbf{R}}$ matrices contained the exact same set of edges as the corresponding $\mathbf{C C}^{\mathbf{M}}$ networks. We used the same thresholding mask for both types of matrices, because our aim is to show the degree of correspondence between the two, subject to identical conditions. Moreover, such a procedure guarantees the same number of edges to be present in both networks, thereby facilitating network comparison (Stam et al., 2014). Importantly however, the weights associated with a given edge could differ in both cases, because in general $\mathbf{C C}^{\mathbf{R}} \neq \mathbf{C C}^{\mathbf{M}}$. For a given threshold $T$, the weighted adjacency matrices resulting from the thresholding procedure are denoted by $\mathbf{A}^{\mathbf{R}, \mathbf{T}}$ and $\mathbf{A}^{\mathbf{M}, \mathbf{T}}$ respectively (see supplementary material S1 for a detailed mathematical definition of those matrices).

For each seizure and time window, we constructed networks defined over a range of thresholds, the latter being given by the $0,10, \ldots, 70,75, \ldots, 95$-th percentiles of the (pooled) elements of $\mathbf{C C}^{\mathbf{M}}$. In the following, we refer to $p_{n}$ as the $n$-th percentile of the pooled elements of $\mathbf{C C}^{\mathrm{M}}$. 


\subsubsection{Construction of Surrogate Functional Networks}

Because for all $(i, j) \in \mathcal{E}$ the CL-algorithm fits the probabilities $P\left(x_{i}, x_{j}\right)$ exactly to their corresponding counterparts $P^{*}\left(x_{i}, x_{j}\right)$ of the (discretized) empirical iEEG data (eq. 2.5), it follows from eq. 2.7 that $c c_{i j}^{M} \approx c c_{i j}^{R}$, with the approximation being solely due to the discretization procedure, whose impact on CC is small (see results). Moreover, the CLalgorithm is based on collecting the edges with largest mutual information edge weight, which in many cases is paralleled by a high value of the corresponding $c c_{i j}^{M}$. To control for the possibility that our results are not just a somewhat trivial consequence of the CLalgorithm collecting the edges with largest edge weight -or equivalently, that the truly inferred $c c_{i j}^{M},(i, j) \notin \mathcal{E}$ do not play a significant role for the results- we have also analyzed two types of surrogate networks, which were constructed in the following way:

Starting from the set of edges defined by $\mathbf{A}^{\mathbf{M}, \mathbf{T}}$, the first type of surrogate networks was created by preserving those edges and weights that were found by the CL-algorithm and "survived" the subsequent thresholding procedure. The remaining edges of $\mathbf{A}^{\mathbf{M}, \mathbf{T}}$ in contrast were assigned to random pairs of nodes. In the latter case, the corresponding edge weights were drawn independently from a uniform distribution. We call networks of this form type I surrogate networks, their adjacency matrices are denoted by $\mathbf{A}^{\mathbf{S}_{\mathbf{I}}, \mathbf{T}}$.

The second type of surrogate networks is complementary to the first one, since it is based on preserving the truly inferred edges and weights (i.e. those not found by the CL algorithm) and scrambling the remaining edges of $\mathbf{A}^{\mathbf{M}, \mathbf{T}}$. We call this the type II surrogate networks having adjacency matrices $\mathbf{A}^{\mathbf{S}_{\text {II }}, \mathbf{T}}$.

Hence, the type I\&II surrogate networks allowed us to selectively determine the impact on the results, both of the weighted edges found by the CL-algorithm and those which were truly inferred through Bayesian inference. See supplementary material S1 for a detailed mathematical definition of these networks.

\subsubsection{Analysis And Comparison Of Functional Networks}

Weighted functional networks were analyzed and compared based on five different measures of network connectivity, namely the modularity $M$ (Newman, 2006; Rubinov \& Sporns, 2010), clustering coefficient $C$ (Onnela et al., 2005; Rubinov \& Sporns, 2010), characteristic path length L (Rubinov \& Sporns, 2010), edge density $D$ and the mean edge-weight $\langle W\rangle$. We have chosen these measures, because they signify seizure characteristics that have previously been reported in the literature (Kramer et al., 2008; Schindler et al., 2008; Kramer et al., 2010). $M$ is a measure of the degree by which a network can "be subdivided into,.., clearly delineated and non-overlapping"subnetworks (Rubinov \& Sporns, 2010) and hence gives a measure of how fragmented the total network is. $C$ in turn measures the average degree of "cliquishness" exhibited by the networks nodes. Informally this means that, e.g. in a network of human relationships, $C$ is high if, on average, two good friends (neighboring nodes) of a person (node) are also good friends to each other. $L$ is the shortest path length between two nodes in the (edge-weighted) network, averaged across all node pairs. Finally, $D$ is the number of edges (with weight $>0$ ) in the network divided by the total number of possible 
edges and $\langle W\rangle$ is its literal definition. Measures $M, C$ and $L$ were analyzed using the "Brain Connectivity Toolbox" (Rubinov et al., 2009).

Also, measures $C$ and $L$ were normalized by constants $C^{R}$ and $L^{R}$ respectively; where $C^{R}$ is the expected clustering coefficient of a network with random topology and edge-weights, that preserves the number of nodes and the edge-weight distribution of the network for which

$C$ was computed. $L^{R}$ is an upper bound for the expected characteristic path length for the same type of random networks. In supplementary material S2 and S3 we give a detailed mathematical account for $C^{R}$ and $L^{R}$ respectively.

Except for $M$, each of these measures was applied separately to each nontrivial, connected network component and we refer to the average across all these components, when reporting values for $C / C^{R}, L / L^{R}, D$ and $\langle W\rangle$ (a nontrivial connected network component is a connected network component containing more than one node). Furthermore, when network measures were applied to seizures that were temporally partitioned into epochs then, for each epoch, we report the average of the (component averaged) measures across all networks belonging to the same epoch.

When different network types are compared by those measures, the $\mathbf{A}^{\mathbf{R}, \mathbf{T}}$ networks are defined to represent the "ground truth", although their topological connectivity (given by the set node pairs that make up the edges) was derived from the $\mathbf{A}^{\mathbf{M}, \mathbf{T}}$ networks. Nevertheless, a given $\mathbf{A}^{\mathbf{R}, \mathbf{T}}$ network can still be regarded as the most realistic member of the class of weighted networks that share the same topological connectivity, since its correlation edgeweights are not based on model predictions, but rather are computed directly from the EEG data. This claim holds true, provided the correlations $a_{i j}^{R, T}>0$ are statistically significant. Thus, for any fixed topological connectivity, it is justified to interpret the $\mathbf{A}^{\mathbf{R}, \mathbf{T}}$ network as the best possible edge-weight improvement for its $\mathbf{A}^{\mathbf{M}, \mathbf{T}}$ counterpart -at least for high $T$, where insignificant correlations have been eliminated.

\section{Results}

Figure 1 provides an overview over the different preprocessing methods and subsequent analyses that have been conducted in this paper. In the following subsections, results obtained from the analysis methods are presented. 


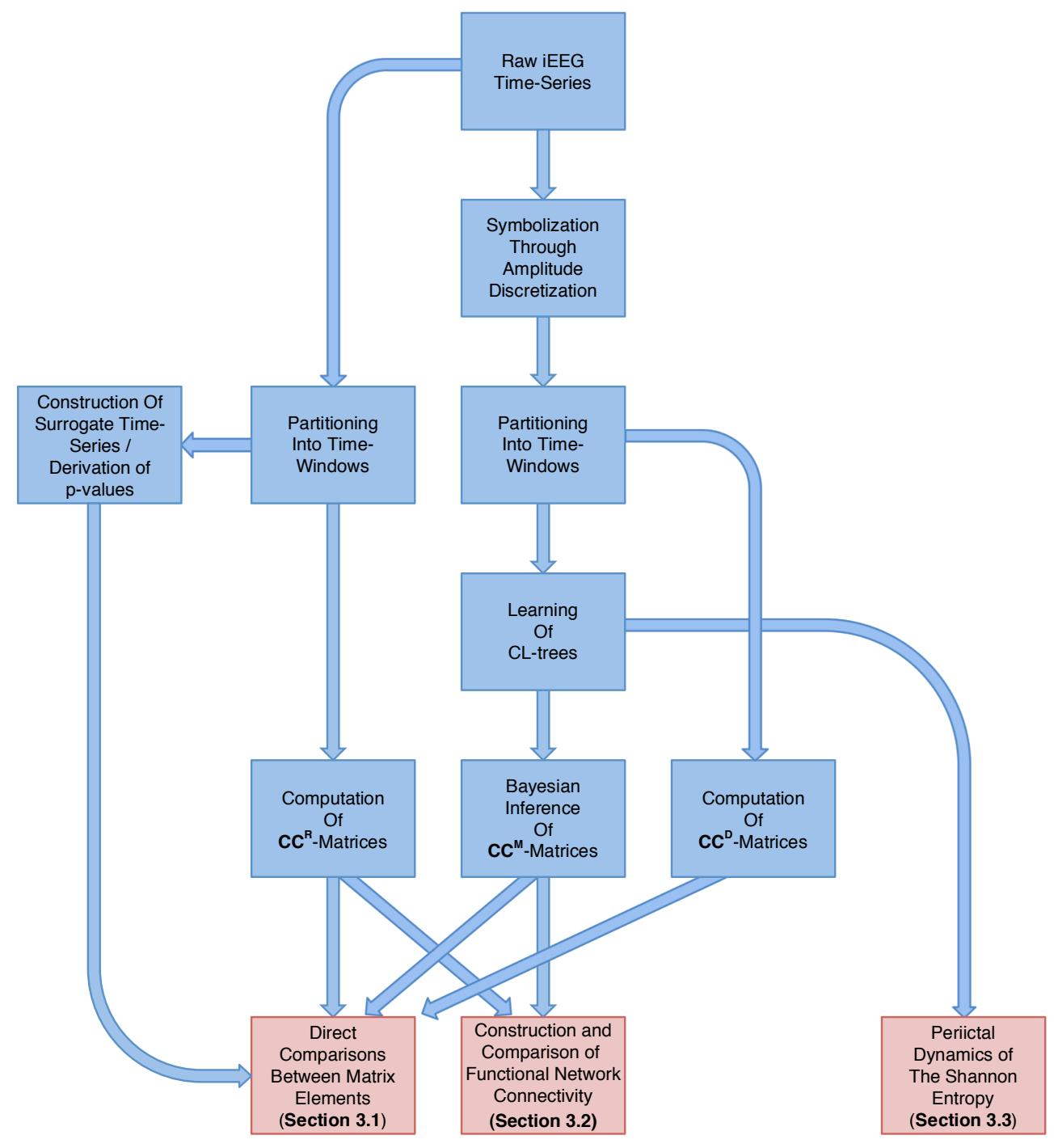

Figure 1: Process Flow of Preprocessing and Analysis Methods Applied to the Raw, Periictal iEEG Time-Series

Blue boxes give preprocessing methods applied before subsequent analysis (red boxes) was performed. Both types of methods are described in the methods section. Input/output relationships between the various methods are depicted by arrows. Results presented in the results section have been obtained from analysis methods. 


\subsection{Direct, Elementwise Comparison of $\mathrm{sCC}^{\mathrm{R}}, \mathrm{sCC}^{\mathrm{D}}$ and $\mathrm{sCC}^{\mathrm{M}}$ Matrices}

In this section we compare the matrices of signed Pearson correlation-coefficients, as constructed from the raw data $\left(\mathbf{s C C}^{\mathbf{R}}\right)$, from the discretized data $\left(\mathbf{s C C}^{\mathbf{D}}\right)$, and from the CL-tree model $\left(\mathbf{s C C}^{\mathbf{M}}\right)$ (see section 2.4.1). We also examine the relationship between matrix elements $s C C_{i j}^{M}$ and the statistical significance of their counterparts $s C C_{i j}^{R}$, which was assessed by the associated p-values $p_{i j}$ (section 2.4.3).

Our first step was to investigate the impact of the amplitude discretization procedure (see section 2.2) on the Pearson correlation coefficient matrix. For this analysis, results were obtained from a single representative seizure (figure 2), which can be carried over to the whole seizure database (not shown). Figure 3 shows a direct comparison between the $\mathbf{s C C}^{\mathbf{R}}$ and $\mathbf{s C C}^{\mathbf{D}}$ matrices, extracted from the seizure data of figure 2 for preictal (first and second row), ictal (third row) and postictal (bottom row) time points. A good match between the two can be observed, which is surprising, given the rather coarse level of discretization using $m=6$ bins only. However, the match is slightly dependent on the magnitude of the $s c c_{i j}^{R}$ values, with $s c c_{i j}^{D}>s c c_{i j}^{R}$ for $s c c_{i j}^{R}<0$ and $s c c_{i j}^{D}<s c c_{i j}^{R}$ for $s c c_{i j}^{R}>0$. In other words, the discretization procedure weakly dampens strong (anti)correlations. However, as we will see later, this had a neglectable effect on the functional networks derived from the CL-trees.

Given the close similarity between the two types of matrices, is this match preserved after the $\mathbf{s C C}^{\mathbf{D}}$ matrices have been swapped with their $\mathbf{s C C}^{\mathbf{M}}$ counterparts? To give an answer to that question, we first replotted figure 3 after the matrix swap (figure 4). We can see that correlation damping is also present for the $\mathbf{s C C}^{\mathbf{M}}$ matrices, but its dependence on $s c c_{i j}^{R}$-magnitude is of more complicated nature and expressed more strongly compared to the $\mathbf{s C C}^{\mathbf{D}}$ matrices. Clearly, when ranking correlations by approximation accuracy, it is the large magnitude correlations $\left|s c c_{i j}^{R}\right| \rightarrow 1$ that are approximated best by the corresponding $s c c_{i j}^{M}$ elements, whereas the opposite holds true for the $s c c_{i j}^{D}$ elements in figure 3. Quantitatively however, for $\left|s c c_{i j}^{R}\right| \rightarrow 1$ the degree of approximation is comparable in both cases. Conversely, small-magnitude correlations $\left|s c c_{i j}^{R}\right| \lesssim 0.4$ are set to values $\left|s c c_{i j}^{M}\right| \approx 0$ by the CL-model, in marked contrast to the corresponding $s c c_{i j}^{D}$ correlations. This effect is most obvious in the right column of figure 4, due to the apparent clusters of $s c c_{i j}^{M}$-values around zero, but can also be observed by the reduced overall brightness of the $\mathbf{s C C}^{\mathrm{M}}$ matrices in the middle column.

Hence, the CL-algorithm seems to produce a sparse representation of the iEEG correlations, by retaining the large-magnitude correlations $\left|s c c_{i j}^{R}\right| \rightarrow 1$, while filtering out (setting close to zero) the smaller ones with $\left|s c c_{i j}^{R}\right| \lesssim 0.4$. Furthermore, it may be concluded from figure 4 (right column) that this behavior is unlikely to be associated with statistical significance of the $s c c_{i j}^{R}$ correlations, because the distribution of corresponding p-values $p_{i j}$ seems to be a function of $s c c_{i j}^{R}$ only. Consequently its dependency on $s c c_{i j}^{M}$ seems to be mediated exclusively through the $s c c_{i j}^{R}$ correlations. 


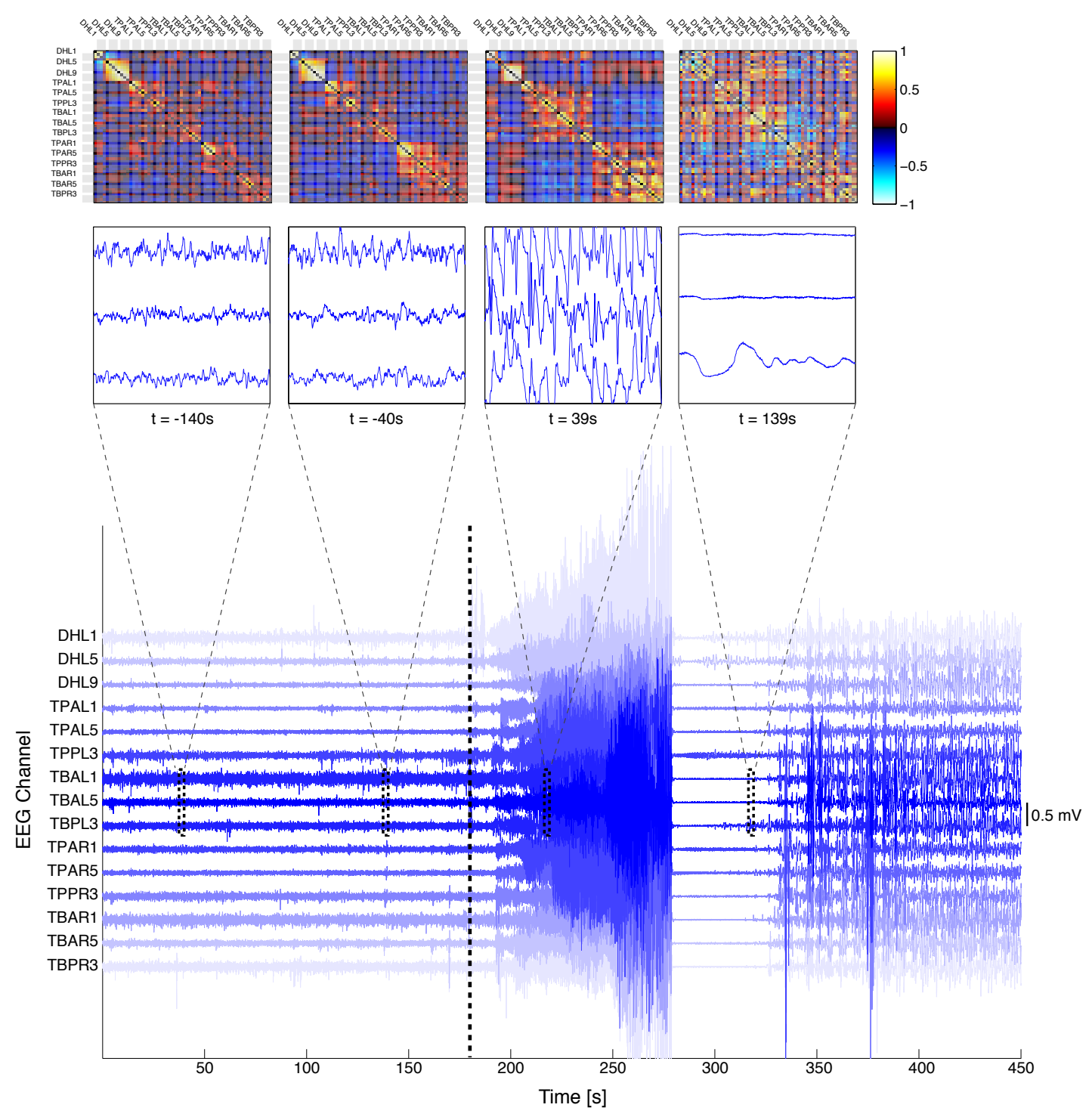

Figure 2: Representative Periictal iEEG Data

Bottom: Periictal time course of the raw voltage signal from example electrodes of patient 7 , seizure 1 (see table 1 ). For clarity only 15 out of 60 total electrodes are shown, whose individual voltage traces are drawn in different shades of blue. Brain locations of the electrodes are indicated on the y-axis (see glossary below for abbreviations). Seizure onset is marked by the vertical black, dashed line, seizure offset corresponds to the abrupt stoppage of strong oscillatory activity around $t=279 \mathrm{~s}$.

Middle row: Zoomed voltage time courses at different time points during evolution of the seizure. Shown are the zoomed sections indicated by the respective dashed boxes in the bottom plot, which correspond to partitioning windows of length $2.5 \mathrm{~s}$. Time point $t$ during seizure evolution is given below each plot and is defined by the ending time of the respective partitioning window relative to seizure onset (seizure onset at $t=0$ ).

Top row: Raw, signed correlation matrices $\mathbf{s C C}^{\mathbf{R}}$ extracted from the full data set of 60 electrodes during the respective partitioning window. Electrodes spared by transparent grey bars are named and correspond to the electrodes shown in the bottom plot.

Glossary of electrode positions: $D H$ : depth hippocampus, $T P / T B$ : temporal polar/basal, $P$ : Posterior, $A$ : Anterior, $L / R$ : Left/Right hemisphere. 

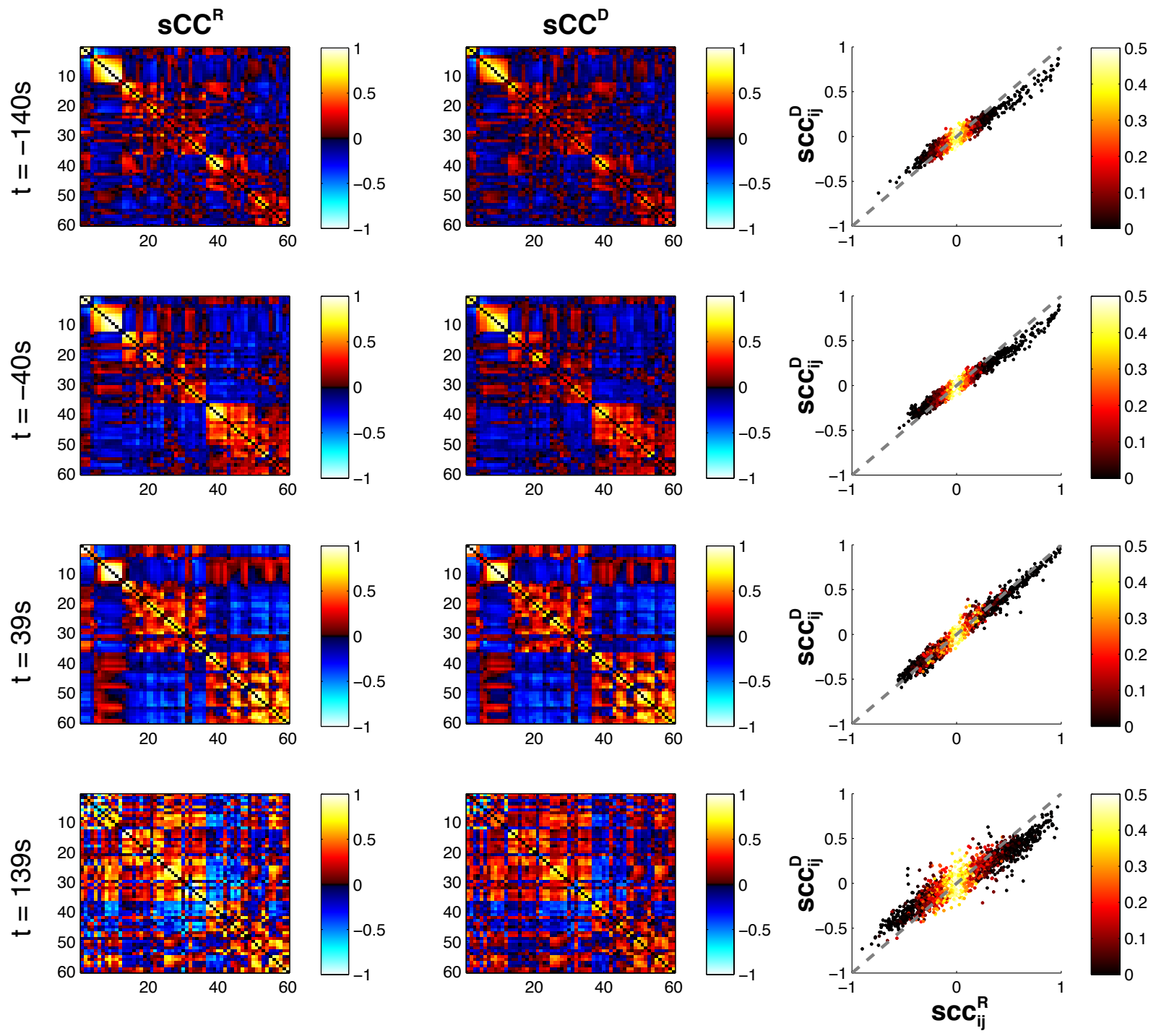

Figure 3: Similarity Between Individual $\mathbf{s C C}^{\mathbf{R}}$ and $\mathbf{s C C}^{\mathbf{D}}$ Matrices Extracted from the Seizure Data Shown in Fig. 2.

Left column: Color-coded matrices $\mathbf{s C C}^{\mathbf{R}}$ for different periictal time points. Time $t$ with respect to seizure onset is indicated on the left (with negative values corresponding to preictal time points, see fig. 2). Central column: The $\mathbf{s C C}^{\mathbf{D}}$ matrices of the corresponding time points. Right column: Matrix elements $s c c_{i j}^{R}$ (x-axis) plotted against their corresponding elements $s c c_{i j}^{D}$ (y-axis). Color indicates p-values $p_{i j}$ associated with elements $s c c_{i j}^{R}$. Grey dashed line is the space diagonal corresponding to points $s c c_{i j}^{M}=s c c_{i j}^{R}$. 

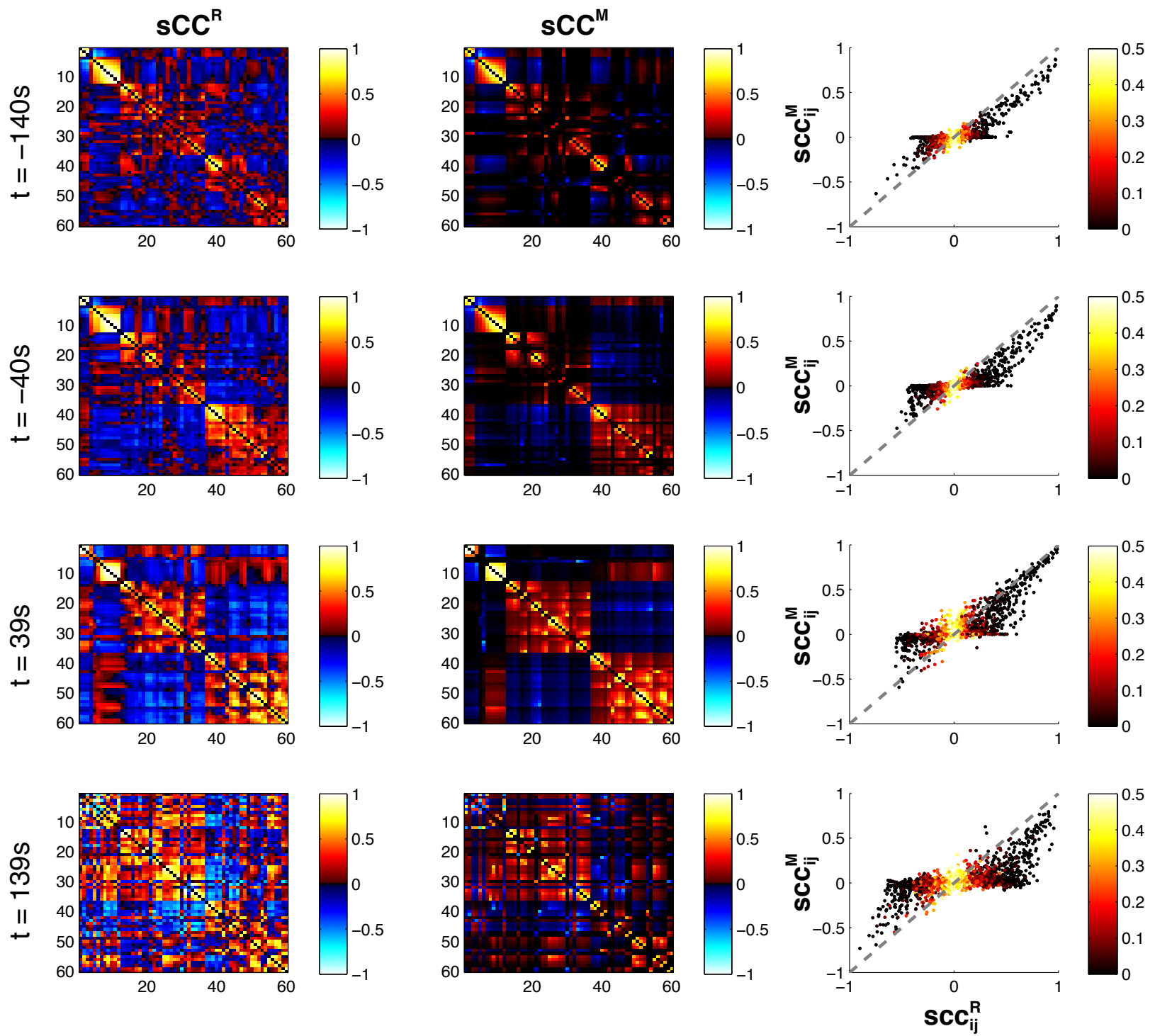

Figure 4: Similarity Between Individual $\mathbf{s C C}^{\mathbf{R}}$ and $\mathbf{s} \mathbf{C C}^{\mathbf{M}}$ Matrices Extracted from the Seizure Data Shown in Fig. 2.

Left column: Color-coded matrices $\mathbf{s} \mathbf{C C}^{\mathbf{R}}$ for different periictal time points. Time $t$ with respect to seizure onset is indicated on the left (with negative values corresponding to preictal time points). Central column: The $\mathbf{s} \mathbf{C C}^{\mathbf{M}}$ matrices of the corresponding time points. Right column: Matrix elements $s c c_{i j}^{R}$ (x-axis) plotted against their corresponding elements $s c c_{i j}^{M}$ (y-axis). Color indicates p-values $p_{i j}$ associated with elements $s c c_{i j}^{R}$. Grey dashed line is the space diagonal corresponding to points $s c c_{i j}^{M}=s c c_{i j}^{R}$. 
Our next question to ask was, whether these relationships observed between individual $\mathbf{s C C}^{\mathbf{R}}$ and $\mathbf{s} \mathbf{C C}^{\mathbf{M}}$ matrices constitute more general phenomena, that generalize across our whole seizure database. More specifically, we examined (a) the average matching accuracy between the two types of matrices, (b) the sparsity of $\mathbf{s C C}^{\mathbf{M}}$ through the neglect of small correlations and (c) the dependency between correlations $s C C_{i j}^{M}$ and the statistical significance of their $s C C_{i j}^{R}$ counterparts.

Examinations (a) and (b) were conducted by the procedure described in section 2.4.2. That is, based on the graphs in the right columns of figures $3 \& 4$, we formed the level sets $s C C_{k}^{R}$ for each correlation level $k \in\{-0.94, \ldots, 0, \ldots, 0.94\}$. The means $\left\langle s C C_{k}^{R}\right\rangle$ and standard deviations $\sigma\left(s C C_{k}^{R}\right)$ then respectively served as measures of matching accuracy and sparsity. Figure 5 shows the results. Firstly, an excellent match between $\left\langle s C C_{k}^{R}\right\rangle$ and $k$ can be observed in (a), which is independent of seizure epoch and indicates a close average correspondence between the $s c c_{i j}^{R}$ and $s c c_{i j}^{M}$ elements. Although there is a bias in fairly large intervals around $k=0.5$ and $k=-0.25$ respectively, its numerical extent is small. Most likely the bias is caused by the damping of large correlations due to the amplitude discretization procedure (cf. figure 3). For very large $k \rightarrow 1$ in turn, the bias vanishes, possibly because very large correlations are confined mostly to those correlations which are trivially matched through the edge-set of the CL-tree (see section 2.3.2).

Secondly, in figure $5 \mathrm{~b}$ there is a prominent peak of the sparsity $\sigma\left(s C C_{k}^{R}\right)$ at $k=0$, which falls off monotonically for $|k| \rightarrow 1$. Thus, we may conclude that for the type of considered seizure data, the CL-model is generally able to find accurate, unbiased but sparse representations of signed Pearson correlation-coefficient matrices. Whereas matching accuracy $\left\langle s C C_{k}^{R}\right\rangle$ was found to be independent of seizure epoch, the sparsity measure $\sigma\left(s C C_{k}^{R}\right)$ increases slightly but steadily towards seizure end. 

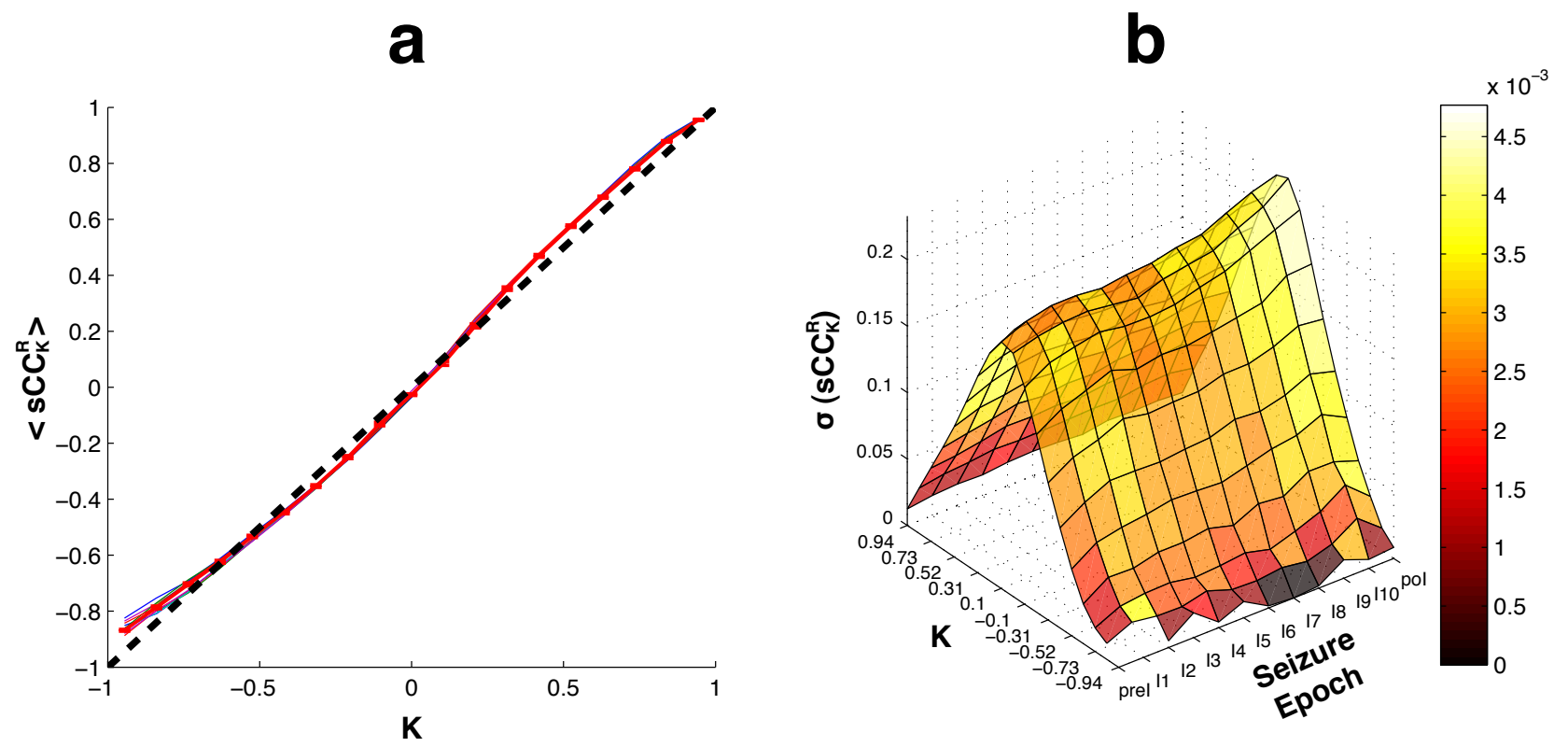

Figure 5: High Average Matching Accuracy and Sparsity of $\mathbf{s} \mathbf{C C}^{\mathbf{M}}$ Matrices a) Seizure averaged $\left\langle s C C_{k}^{R}\right\rangle$ vs. $k \in\{-0.94, \ldots, 0, \ldots, 0.94\}$. The thin, colored curves correspond to the different seizure epochs. Representative epoch $\mathrm{I}_{10}$ is drawn as a thick, red line, whose errorbars illustrate s.e.m. for the averaging across seizures. Errorbars of the other curves have been omitted for clarity. The thick, dashed line is the space diagonal indicating all those points for which the average $\left\langle s C C_{k}^{R}\right\rangle$ is equal to $k$.

b) Seizure averaged $\sigma\left(s C C_{k}^{R}\right)$ vs. $k$ and seizure epoch. Surface color indicates s.e.m. for the averaging across seizures.

A question that naturally arises from these findings is, whether large $s c c_{i j}^{M}$ values imply statistical significance of their $s c c_{i j}^{R}$ counterparts (examination (c) above). Conversely, $s c c_{i j}^{M}$ correlations with values close to zero could signify a reduction in type I errors, by suppressing those $s c c_{i j}^{R}$ for which the hypothesis of being random correlations -due to finite size of the partitioning windows- cannot be excluded (see section 2.4.3). If such a relationship between the $\mathbf{s C C}^{\mathbf{M}}$ and statistical significance was present, a sparse representation of $\mathbf{s} \mathbf{C C}^{\mathbf{R}}$ by $\mathbf{s C C}^{\mathbf{M}}$ would indicate a concentration of the CL-model on those genuine correlations, for which randomness can be excluded with high probability. Although in principle there is nothing in the definition of the CL-algorithm that distinguishes random from nonrandom correlations (apart from the latters' correspondence to large correlation- and thus also mutual information values), such a distinction could still be an epiphenomenon of the CL-algorithm when applied to periictal iEEG data. If in figure 4, right column for example, the iEEG data was such that for most correlations $\left|s c c_{i j}^{R}\right| \lesssim 0.4$ randomness cannot be excluded (through large associated p-values $p_{i j}$ ), the CL-algorithm would have set their $s c c_{i j}^{M}$ partners to values close to zero, thereby having eliminated critical correlations indirectly.

To investigate this possibility more generally for seizures from our database, we examined 
the distribution of p-values at each correlation level $k$, using the procedures described in section 2.4.3. Figure 6a,b shows that large correlations $\left|s c c_{i j}^{M}\right|$ are indeed highly unlikely to be associated with random $s c c_{i j}^{R}$ partners, as for $|k| \rightarrow 1$ the mean and standard deviation of the sets $P_{k}$ both shift towards zero, irrespective of seizure epoch. On the other hand, for $k=0$ the null hypothesis $H_{0}$ of correlations $s c c_{i j}^{R} \in s C C_{k=0}^{R}$ being random correlations is unlikely to hold either, since across epochs the means $\left\langle P_{k=0}\right\rangle$ are consistently lower than 0.25 , the mean of a uniform distribution on the interval $[0,0.5]$. This impression is confirmed by a t-test on those means which, for each epoch, yielded vanishingly small p-values that were below floating-point accuracy of the computer. Finally, histograms of $P_{k=0}$ reveal distributions that are significantly different from a uniform distribution (figure 6c). Taken together, $H_{0}$ can be safely refuted by the evidence.

To summarize this section, we have shown that correlation matrices $\mathbf{s C C}^{\mathbf{M}}$ inferred from the CL-model yield accurate, yet sparse versions of their "true" empirical counterparts $\mathbf{S C C}^{\mathbf{R}}$. In this context the term "accurate, yet sparse" refers to the fact that small correlations $\left|s c c_{i j}^{R}\right|$ are mapped to even smaller partners $\left|s c c_{i j}^{M}\right| \approx 0$, whereas the large $\left|s c c_{i j}^{R}\right|$ are faithfully preserved by the CL-model. However, although large correlations $\left|s c c_{i j}^{M}\right| \rightarrow 1$ are not associated with random correlations $s c c_{i j}^{R}$, the converse does not hold; small correlations $\left|s c c_{i j}^{M}\right| \rightarrow 0$ do generally not imply random $s c c_{i j}^{R}$-partners. 
a

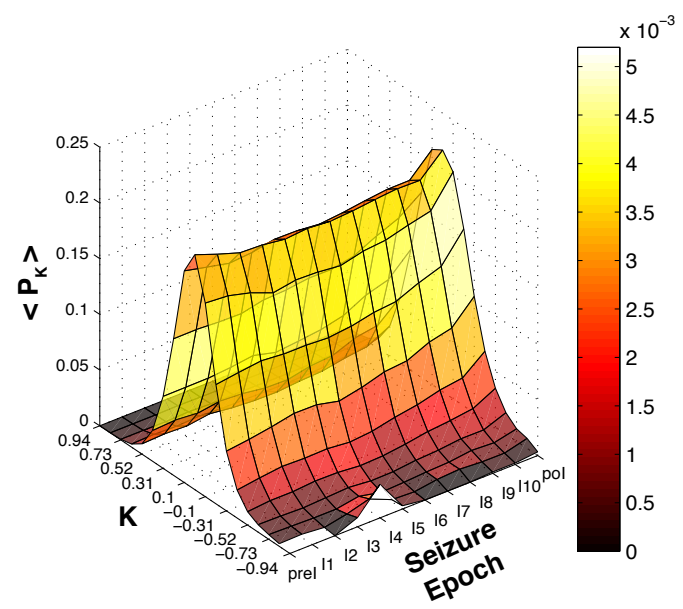

C

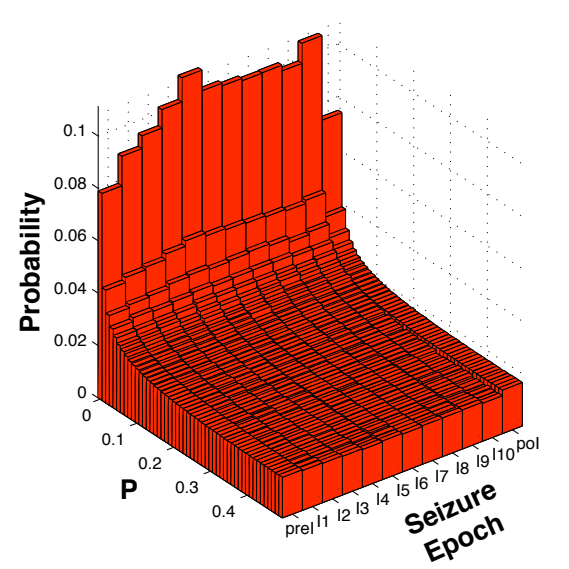

b

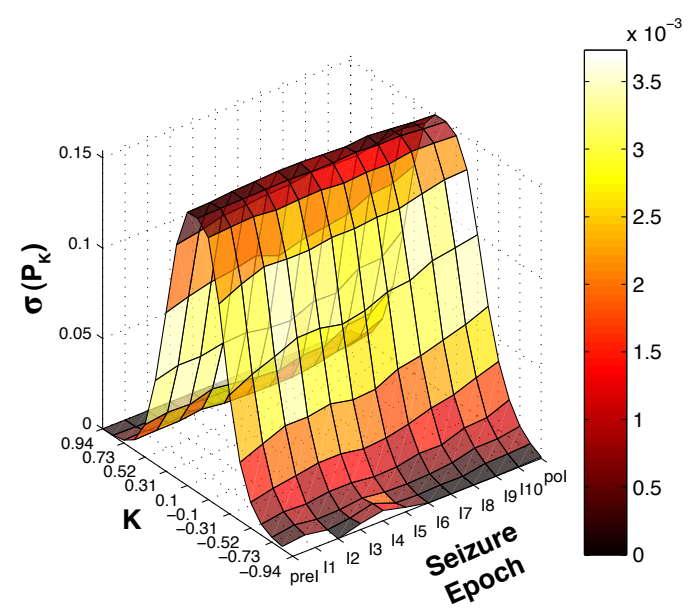

Figure 6: Large CL-Correlations $\left|s c c_{i j}^{M}\right|$ Signify Nonrandom Empirical Correlations $s c c_{i j}^{R}$. Small $\left|s c c_{i j}^{M}\right|$ Do Not Signify Randomness of $s c c_{i j}^{R}$.

a) Seizure averaged mean of $P_{k}$ vs. $k$ and seizure epoch. For a given $k$, seizure epoch and seizure the mean was taken across the union of all sets $P_{k}$, whose corresponding partitioning windows belonged to the given epoch. The result was then averaged again across all seizures, yielding $\left\langle P_{k}\right\rangle$ on the z-axis. Surface color indicates s.e.m. for the averaging across seizures.

b) Seizure averaged standard deviation of $P_{k}$ vs. $k$ and seizure epoch. For a given $k$, seizure epoch and seizure the mean was taken across all standard deviations of $P_{k}$, whose corresponding partitioning windows belonged to the given epoch. The result was then averaged again across all seizures, yielding $\left\langle P_{k}\right\rangle$ on the z-axis. Surface color indicates s.e.m. for the averaging across seizures.

c) Probability distribution of the p-values $p$ in the union of all $P_{k=0}$ from all seizures and partitioning windows that belong to the same seizure epoch. The distribution deviates significantly from a uniform distribution. 


\subsection{Comparison Of $\mathrm{CC}^{\mathrm{R}}$ And $\mathrm{CC}^{\mathrm{M}}$ Matrices On The Basis Of The Functional Networks They Induce}

In this section we show the correspondence between the raw $\left(\mathbf{C C}^{\mathbf{R}}\right)$ and the CL-derived $\left(\mathbf{C C}^{\mathbf{M}}\right)$ unsigned correlation-coefficient matrices, on the basis of their induced functional networks $\mathbf{A}^{\mathbf{R}, \mathbf{T}}$ and $\mathbf{A}^{\mathbf{M}, \mathbf{T}}$ respectively (section 2.5.1). Correspondence is shown based on the five measures of network connectivity described in section 2.5.3. In the discussion we will elaborate on how connectivity of these networks displays the key features that have previously been reported in a variety of studies for periictal functional networks.

Recalling our results from the previous section, the CL-algorithm apparently filters out small correlations, but approximately preserves the larger ones. Hence, we were interested to see how this effect influences the properties of functional networks $\mathbf{A}^{\mathbf{R}, \mathbf{T}}$ and $\mathbf{A}^{\mathbf{M}, \mathbf{T}}$, because the transition from mismatch in correlation edge-weights to corresponding mismatch in functional network connectivity is nontrivial.

To get a notion of their connectivity, figure 7a shows the structure of some of the $\mathbf{A}^{\mathbf{M}, \mathbf{T}}$ networks that were derived from our representative seizure of figure 2. For this patient, apparently, nearby electrodes belonging to the same brain area are clustered into densely intraconnected subnetworks, that are in comparison only weakly connected to subnetworks from other areas. During the seizure $(t=39)$ this effect is best visible and suggests a high degree of modularity. Clearly, most of the network measures we consider in this study, namely the modularity $M$, normalized clustering coefficient $C / C^{R}$, normalized characteristic path length $L / L^{R}$ and mean edge-weight $\langle W\rangle$, do not only depend on the topology of a given network, but also on the specific weights of each of the networks edges. A priori it is not clear to what extent those measures are influenced by even small differences/errors between the edge-weights of $\mathbf{A}^{\mathbf{M}, \mathbf{T}}$ and $\mathbf{A}^{\mathbf{R}, \mathbf{T}}$ networks.

However, figure $7 \mathrm{~b}$ suggests such errors to have a surprisingly small effect on the time courses of $M, C / C^{R}$ and $L / L^{R}$, when the seizure is again divided temporally into a set of 12 epochs $\left\{\operatorname{prI} I, I_{1}, \ldots, I_{10}, p o I\right\}$. In the following we will term such a temporal evolution of a given measure through the different seizure epochs the "epoch course" of that measure. For $\mathbf{A}^{\mathbf{M}, \mathbf{T}}$ networks, the epoch courses of $M, C / C^{R}$ and $L / L^{R}$ (blue lines) track very closely the hilly profile of the corresponding courses for the $\mathbf{A}^{\mathbf{R}, \mathbf{T}}$ networks (red lines), which consists of a net increase of the respective measure towards seizure midtime, followed by an equivalent net decrease towards seizure end. In fact, for $M, C / C^{R}$ and $L / L^{R}$ the relative error between the blue and the red lines is smaller than the relative error for the mean edge-weight $\langle W\rangle$. Thus, the weight-dependent connectivity of $\mathbf{A}^{\mathbf{M}, \mathbf{T}}$ networks (when measured by $M, C / C^{R}$ and $L / L^{R}$ ) seems to be highly robust against the kind of edge-weight mismatch that is introduced by amplitude discretization and the CL-model. 


\section{a}
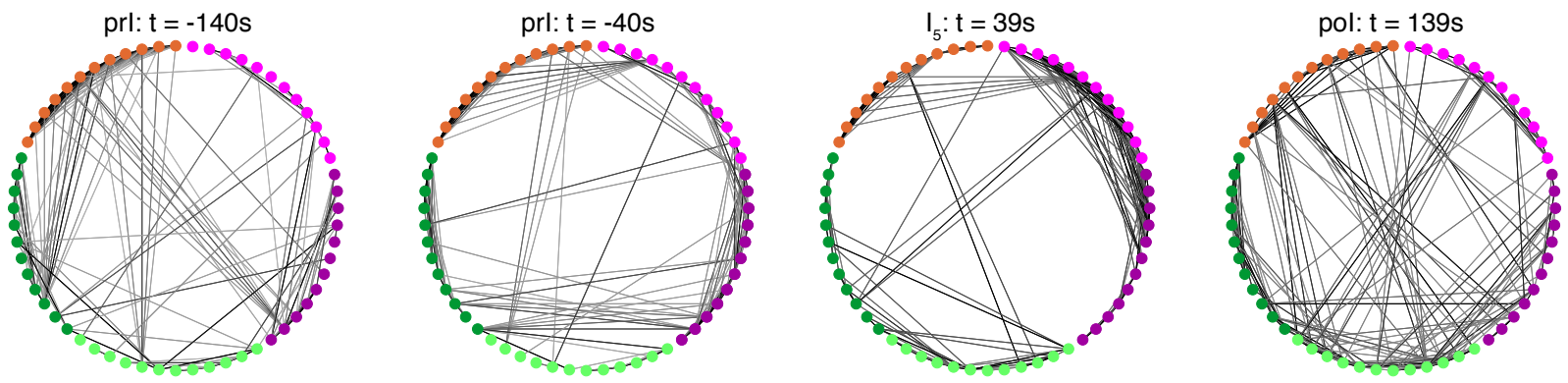

- Deep Hippocampus Left (DHL*)

- Temp. Polar Left $\left(T P^{*} L^{*}\right)$

- Temp. Basal Left $\left(\mathrm{TB}^{*} \mathrm{~L}^{*}\right)$

- Temp. Polar Right $\left(T^{\star} R^{\star}\right)$

b
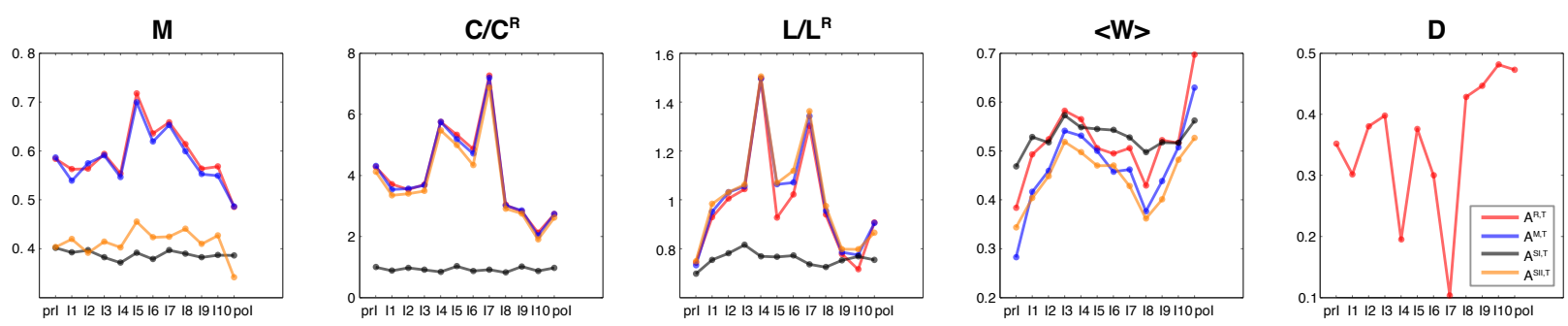

Figure 7: Example Periictal Evolution of Functional Network Connectivity

a) Connectivity of $\mathbf{A}^{\mathbf{M}, \mathbf{T}}$ networks (with $T=p_{90}$ ) at different periictal time points $t$ for the representative seizure of figure 2. $t$ is measured with respect to seizure onset $(t=0)$, its corresponding seizure epochs are indicated to the left of $t$. Grey shadings of edges between nodes are proportional to the edge-weight $c c_{i j}^{M}$ of the corresponding electrode pair (the larger the weight, the darker the shading). Node colors indicate the approximate location of the respective electrode.

b) Periictal evolution of network connectivity for each type of network. Shown are the time courses through the different epochs of the seizure in (a) for the five measures of network connectivity we consider in this study; $M$ : Modularity,$C / C^{R}$ : Normalized clustering coefficient, $L / L^{R}$ : Normalized characteristic path length, $\langle W\rangle$ : Mean edge-weight, $D$ : Edge density. Line color indicates the type of considered network (see legend in the rightmost plot).

Are these findings reproducible in a large set of seizures from various patients? To answer this question we applied the analysis of figure $7 \mathrm{~b}$ to our whole seizure database. For each network measure and periictal epoch, summary statistics were formed by; first, averaging the measure in question for all networks of a given seizure that belonged to the same epoch and secondly by averaging the result again across the whole database. Furthermore, we considered a range of thresholds $T \in\left\{p_{0}, p_{30}, p_{60}, p_{75}, p_{90}, p_{95}\right\}$.

Figure 8 shows the resulting epoch courses for each of the measures and thresholds. The 
first thing to observe for $\mathbf{A}^{\mathbf{M}, \mathbf{T}}$ networks is convergence of each measures epoch course (blue lines) to the corresponding epoch course of the $\mathbf{A}^{\mathbf{R}, \mathbf{T}}$ networks (red lines), when $T \rightarrow p_{100}$. This holds true except for the mean edge-weight $\langle W\rangle$, which is, once again, most likely caused by amplitude discretization of the raw data, because this artifact exerts its strongest effect on the large correlations and becomes the dominant source of error for $T \rightarrow p_{100}$ (cf. figures 3, 4, 5a and main text of section 3.1). However, for $T \geq p_{75}$ the relative error in $\langle W\rangle$ is again larger than the corresponding error in $M, C / C^{R}$ and $L / L^{R}$. Therefore, as for the representative seizure, errors regarding $\langle W\rangle$ had a remarkably little effect on the other connectivity measures, whose epoch courses converge accordingly. In other words, although there is some space for improving the edge-weights of $\mathbf{A}^{\mathbf{M}, \mathbf{T}}$ networks, there is virtually no space for improving their connectivity w.r.t. $M, C / C^{R}$ and $L / L^{R}$. Because this finding is based on a large set of 93 seizures, we propose it to be a general attribute of the CL-model when applied to periictal iEEG data.

Likewise, figure 8 shows that the hilly profiles the epoch courses of $M, C / C^{R}$ and $L / L^{R}$ converge to are not exclusive properties of particular seizures only, they are rather preserved across the seizure database. As we will elaborate further in the discussion, such profiles (together with the late-increasing profile of $D$ ) are key features of periictal functional networks that have been widely discussed in the literature.

A third aspect to observe in figure 8 is the fact that convergence of the epoch courses cannot be solely explained by the correlations that are trivially matched by the CL-algorithm. This is because the epoch courses of the $\mathbf{A}^{\mathbf{S}_{\mathbf{I}}, \mathbf{T}}$-networks are markedly different from those of the corresponding $\mathbf{A}^{\mathbf{M}, \mathbf{T}}$-networks. The epoch courses of $\mathbf{A}^{\mathbf{S}_{\mathbf{I I}}, \mathbf{T}}$-networks in contrast are in most cases very similar to those of the $\mathbf{A}^{\mathbf{M}, \mathbf{T}}$-networks but, for $M,\langle W\rangle$ and large $T$, diverge from them and hence also from the epoch courses of the $\mathbf{A}^{\mathbf{R}, \mathbf{T}}$-networks.

To better illustrate these different convergence modes, we have further characterized the epoch courses by two quantities: First, for each seizure we took the mean of a given epoch course across all epochs. Second, we computed the Pearson correlation coefficient between the epoch course for $\mathbf{A}^{\mathbf{R}, \mathbf{T}}$-networks and the corresponding courses for $\mathbf{A}^{\mathbf{M}, \mathbf{T}}, \mathbf{A}^{\mathbf{S}_{\mathbf{I}}, \mathbf{T}}$ and $\mathbf{A}^{\mathbf{S}_{\mathbf{I I}}, \mathbf{T}}$-networks. Both quantities, the epoch means and Pearson correlations, were then averaged across all seizures. Figure 9 shows the result. Clearly, the impression suggested by figure 8 is confirmed; For increasing $T$ and any measure apart from $\langle W\rangle$, the mean epoch

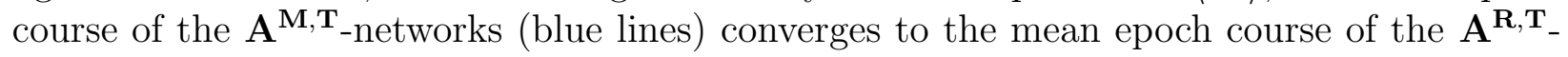
networks (red, dashed space diagonal). This is paralleled by a steady increase in correlation between the two epoch courses, reaching almost 1 for $T=p_{95}$. Conversely, except for the mean edge weight, the epoch courses of $\mathbf{A}^{\mathbf{S}_{\mathbf{I}}, \mathbf{T}}$-networks (black lines) correlate poorly with those of the $\mathbf{A}^{\mathbf{R}, \mathbf{T}}$-networks. Contrary to intuition this is true even for high $T$, for which

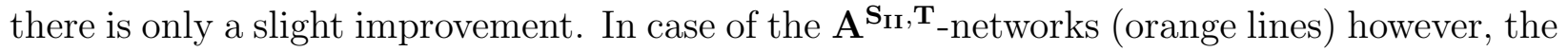
$C / C^{R}$ and $L / L^{R}$ epoch courses are very similar to those of the $\mathbf{A}^{\mathbf{R}, \mathbf{T}}$ networks, as indicated by both, mean epoch courses that converge to their respective space diagonal and Pearson correlations that reach almost 1 for high $T$. For $M$ and $\langle W\rangle$ in contrast, the epoch courses start to diverge for high $T$, as reflected in decreasing correlations and divergence from the space diagonals. A possible explanation for this finding is the increasing importance of 
CL-matched correlations when $T \rightarrow p_{100}$, because these edge-weights are relatively large in magnitude but randomized for $\mathbf{A}^{\mathbf{S}_{\mathbf{I I}}, \mathbf{T}}$-networks. This may also explain the opposite behavior of $\mathbf{A}^{\mathbf{S}_{\mathbf{I}}, \mathbf{T}}$-networks, which preserve the CL-matched correlations and whose performance for $M, L / L^{R}$ and $\langle W\rangle$ increases slightly when $T \rightarrow p_{100}$.

In summary, figures 8 and 9 clearly show that for high thresholds $T, \mathbf{A}^{\mathbf{M}, \mathbf{T}}$-networks are capable of reproducing the connectivity of their corresponding $\mathbf{A}^{\mathbf{R}, \mathbf{T}}$-networks. While the quality of this reproduction improves steadily for increasing $T$, this is not the case for either type of surrogate network. Thus, it is the combined effect of both types of correlations $c c_{i j}^{M}$, the ones trivially matched by the CL-algorithm and the ones truly inferred through Bayesian inference, that is needed for a reproduction of functional network connectivity. 

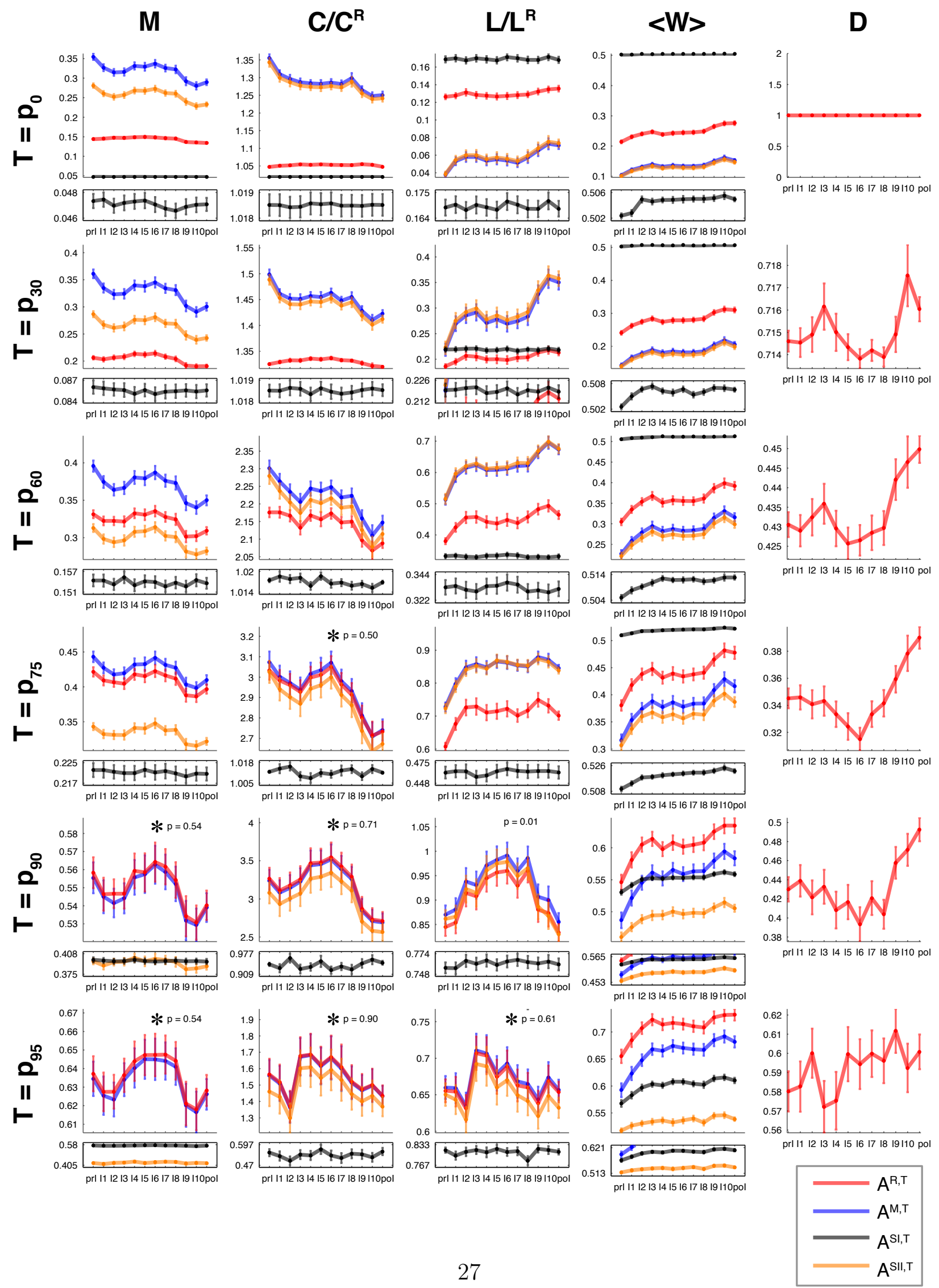
Figure 8: Average Epoch Courses of Connectivity Measures Taken from the Different Functional Network Types

Shown is the evolution across seizure epochs of the modularity (first column), clustering coefficient (second column), characteristic path length (third column), edge density (fourth column) and mean edge weight (last column), averaged across all seizures from the database. Rows correspond to the results for functional networks defined by the different thresholds $T \in\left\{p_{0}, p_{30}, p_{60}, p_{75}, p_{90}, p_{95}\right\}$. Red, blue, black and orange lines give results for $\mathbf{A}^{\mathbf{R}, \mathbf{T}}, \mathbf{A}^{\mathbf{M}, \mathbf{T}}, \mathbf{A}^{\mathbf{S}_{\mathbf{I}}, \mathbf{T}}$ and $\mathbf{A}^{\mathbf{S}_{\mathbf{I I}}, \mathbf{T}}$ networks respectively. Error bars denote s.e.m. for the mean across seizures. The small diagrams below the large ones show a zoom-in of the epoch courses for the $\mathbf{A}^{\mathbf{S}_{\mathbf{I}}, \mathbf{T}}$ or $\mathbf{A}^{\mathbf{S}_{\mathbf{I I}}, \mathbf{T}}$ networks, for their respective network measures may be too different from the ones of the $\mathbf{A}^{\mathbf{R}, \mathbf{T}}$ and $\mathbf{A}^{\mathbf{M}, \mathbf{T}}$ networks. Stars denote cases where the red and blue curves were not significantly different at the $5 \%$ level (two-way ANOVA, factors being network type and epoch, no correction for multiple comparisons (maximally conservative), p-values as indicated). 

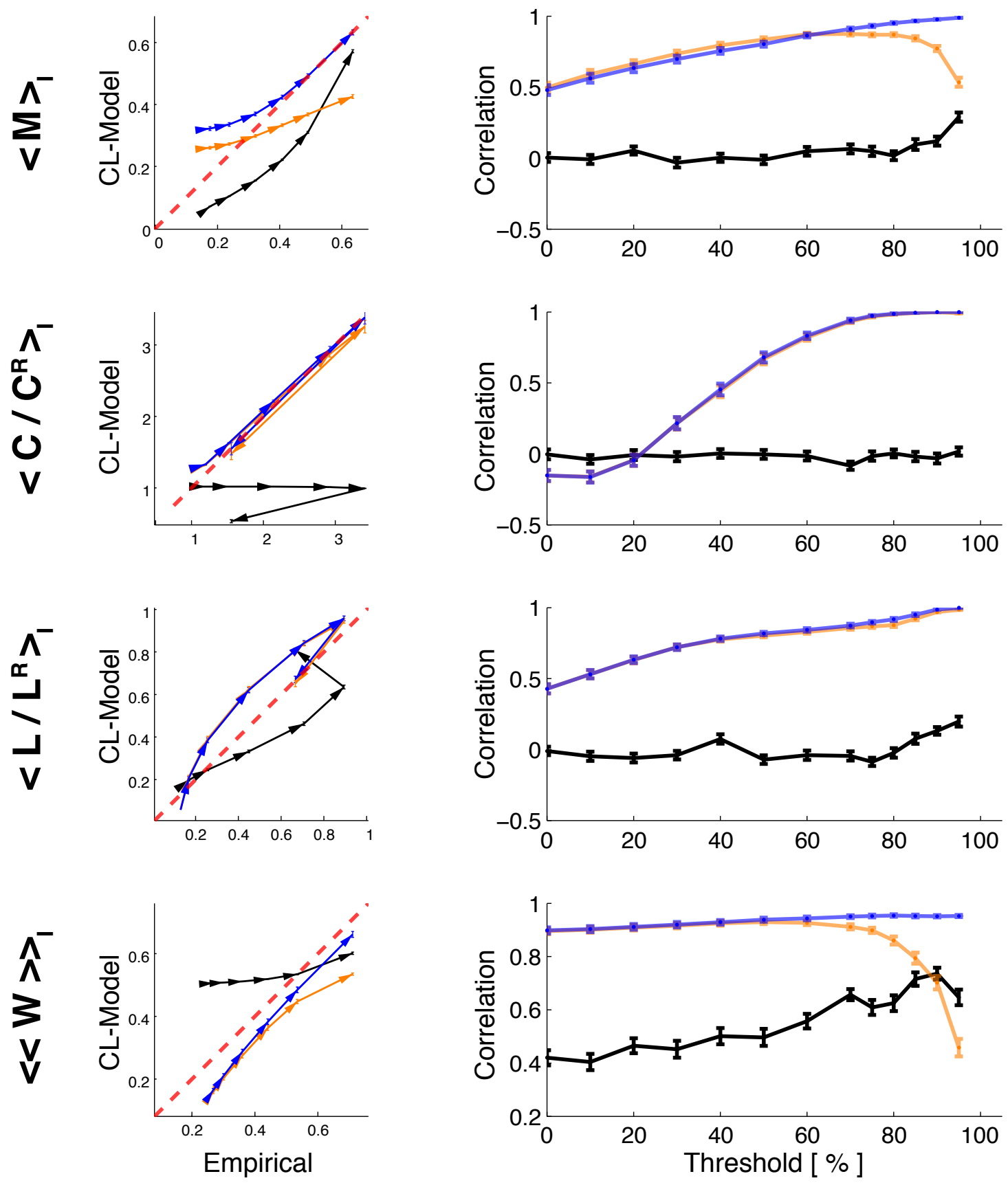

Figure 9: Convergence Modes of Epoch Courses from Different Network Types and Measures Left column: Trajectory plots of the averages $\langle M\rangle_{I}$ (top row), $\left\langle C / C^{R}\right\rangle_{I}$ (second row) $\left\langle L / L^{R}\right\rangle_{I}$ (third row) and $\langle\langle W\rangle\rangle_{I}$ (bottom row), where $\langle\cdot\rangle_{I}$ denotes averaging across all 12 seizure epochs (prI, $\mathrm{I}_{1}, \ldots, \mathrm{I}_{10}, \mathrm{poI}$ ). Values along the $\mathrm{x}$-axis were formed by averaging the $\langle\cdot\rangle_{I^{-}}$values for $\mathbf{A}^{\mathbf{R}, \mathbf{T}}$-networks across all seizures, whereas values along the $\mathrm{y}$-axis were formed by the seizure-averaged $\langle\cdot\rangle_{I^{-}}$-values of the corresponding $\mathbf{A}^{\mathbf{M}, \mathbf{T}}$ (blue), $\mathbf{A}^{\mathbf{S}_{\mathbf{I}}, \mathbf{T}}$ (black) and $\mathbf{A}^{\mathbf{S}_{\mathbf{I I}}, \mathbf{T}}$ (orange) networks. Arrows connect points corresponding to neighboring thresholds $T \in\left\{p_{0}, p_{20}, p_{40}, p_{60}, p_{75}, p_{85}, p_{95}\right\}$ and point towards the higher threshold. Red, dashed line shows the space diagonal corresponding to points $x=y$. (vanishingly small) error bars denote s.e.m. for the mean across seizures.

Right column: Threshold dependent Pearson correlation coefficients between the epoch courses of $\mathbf{A}^{\mathbf{R}, \mathbf{T}_{-}}$ networks and the courses of the corresponding $\mathbf{A}^{\mathbf{M}, \mathbf{T}}, \mathbf{A}^{\mathbf{S}_{\mathbf{I}}, \mathbf{T}}$ and $\mathbf{A}^{\mathbf{S}_{\mathbf{I I}}, \mathbf{T}}$ networks. x-axis gives threshold in terms of percentile percent. Color and row definitions as in left column. Error bars denote s.e.m. for the mean across seizures. 


\subsection{Periictal Dynamics of the Shannon Entropy}

In this section we show the temporal, epoch-based evolution of the Shannon entropy, computed analytically for each of a seizures CL-trees (section 2.3.1). In the discussion, we will relate our results to putative functional mechanisms behind this epoch course, as they have emerged in recent years.

A longstanding view in epilepsy research is to regard seizures as states of global hypersynchrony in the EEG, such that the degree of synchronicity between subsets of electrodes stays constantly high during the seizure and is independent of seizure epoch (Schindler et al., 2007). Therefore, we were interested in the periictal evolution of the Shannon entropy $H(\mathbf{x})$, as this measure may be used for characterizing synchronicity in case of EEG data. $H(\mathbf{x})$ is a measure of synchronicity, because the presence of highly synchronous EEG dynamics implies a relatively small number of joint states that the system of electrodes may visit with nonzero probability. Such highly concentrated probability distributions in turn are associated with low entropy.

Hence, for each periictal partitioning window of a given seizure we have computed $H(\mathbf{x})$ for the distribution that is represented by its corresponding CL- tree (ref. eq.2.2). As before, seizures were partitioned into the sequence of epochs $\left\{p r I, I_{1}, \ldots, I_{10}, p o I\right\}$ and summary statistics w.r.t. the whole seizure database were computed separately for each epoch. To allow for averaging across seizures from different patients -which may have been implanted a variable number of iEEG electrodes (ref. table 1)- we have normalized $H(\mathbf{x})$ by the maximally possible entropy $H_{\max }=n \cdot \log m$, i.e. the entropy of a uniform distribution across $m^{n}$ states (where $m=6$ is the number of discretization states and $n$ the number of variables (electrodes).

Figure 10 shows the resulting epoch course of the distribution of normalized entropy values $H(\mathbf{x}) / H_{\max }$. For both, the mean and median normalized entropy, we can observe the hilly profile we have encountered already for $M, C / C R$ and $L / L^{R}$ in the previous chapter. That is, a gradual increase of the mean/median towards $I_{5}$ is followed by a corresponding decrease towards seizure end. Pre and postictal levels of the mean/median normalized entropy are approximately equal. Furthermore, variability of the normalized entropy (when measured as the interquartile range) has an inverse profile, i.e. it decreases towards $I_{5}$ and recovers towards $I_{10}$.

Thus, the presented results are inconsistent with the traditional view regarding synchronicity during epileptic seizures. If the latter were indeed events of temporally constant hypersynchrony, we would have expected a mean/median normalized entropy profile that is (a) minimal and (b) constant during the ictal stages $I_{1}, \ldots, I_{10}$. However, the profile observed is rather suggestive of a scenario, where an initial desynchronization phase that lasts until $I_{5}$ is followed by resynchronization towards seizure end. 


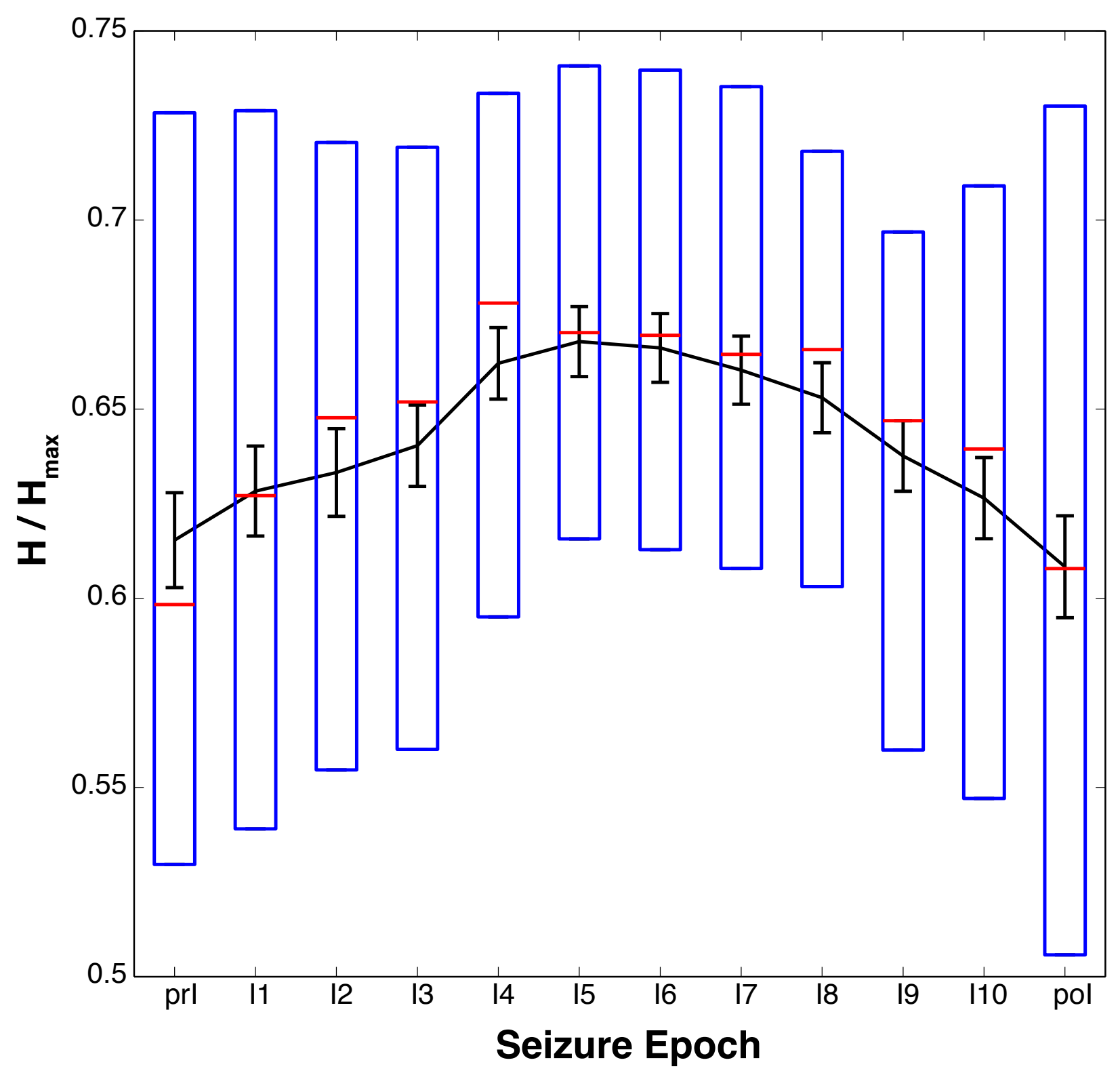

Figure 10: Epoch Course of the Distribution of Normalized Entropy Values.

Shown is the epoch-based evolution of a boxplot representation of the distribution of normalized entropy values $H(\mathbf{x}) / H_{\max }$, ref. eq.2.2, $H_{\max }=n \cdot \log m$, where $m=6$ is the number of discretization states and $n$ the number of variables (iEEG channels). Discretized periictal iEEG data was partitioned into the 12 epochs 'prI' (preictal epoch), ' $\mathrm{I}_{1}, \ldots, \mathrm{I}_{10}$ ' (equidistant ictal epochs) and 'poI (postictal epoch). For each seizure, the normalized entropy values of all CL-trees that belonged to the same epoch were averaged, yielding a single scalar value per epoch and seizure. The blue boxes give the 25th and 75th percentile of these values across seizures and the red line the corresponding median, whereas the black line connects the means across seizures. Error bars represent s.e.m. for the averaging across seizures. 


\section{Discussion \& Conclusion}

The significance of our results with respect to the analysis of periictal iEEG time-series is threefold: Firstly, we have shown that functional networks probabilistically inferred from Chow-Liu (CL) trees excellently reproduce the connectivity of functional networks that were derived empirically from periictal iEEG data. This was assessed with respect to several measures of network connectivity, namely the modularity, clustering coefficient, characteristic path length and edge density. Secondly, the time courses of these measures through the different seizure epochs (which we called "epoch courses" accordingly) show the key features that have previously been reported in the literature (Schindler et al., 2008; Kramer et al., 2010). Thirdly, the Chow-Liu model can be generalized in a straightforward way to become amenable for the modeling of dynamic aspects of time-series, that is, to yield a predictive model also for the temporal dependencies between iEEG time slices.

The results regarding the first point were highly unexpected, because for all thresholds $T$-as they were used to remove network edges based on their their correlation weight, thus defining the topology of functional networks- there was a residual error between the predicted and the empirically derived correlation edge-weights. As we have shown, this error can be attributed partly to our necessary procedure for discretizing the EEG data and is thus unavoidable. However, for high $T$, the relative error between edge-weights from the two types of networks was much larger than the corresponding relative error for the modularity, clustering coefficient and characteristic path length. Hence, for high $T$, the connectivity of functional networks predicted by the CL-model is robust against perturbations in edgeweights. Interestingly, as we will discuss below, it is also the high thresholds that reproduce best the key features of epileptic iEEG time-series reported in (Schindler et al., 2008; Kramer et al., 2010).

Leaving aside correlation networks that were inferred from the CL-trees, we also have to note in this context that functional network analysis could have been applied directly to those trees, in the hope that, as a minimum spanning trees(MSTs), they might have captured the most important features of networks based on mutual information weights. Indeed (Stam et al., 2014) review a variety of studies, where MSTs were able to capture key properties of weighted networks, ranging from main traffic in traffic networks to seizure related topological changes in EEG correlation networks. Analysis of CL-trees per se was beyond the scope of our study however, firstly because most network studies in epilepsy research are based on correlation networks (in contrast to mutual information networks) and secondly because an established "minimum set of MST (network) measures that captures most of the relevant changes in MST morphology" has yet to be found (Stam et al., 2014). For example, analysis of the clustering coefficient is a standard procedure in the field, but it is not meaningful for trees, as this coefficient is always zero in this case, regardless of edge weight distribution and tree topology.

Regarding the second point above, recent results have indicated that epileptic seizures are characterized, up and until seizure mid-time, by a fragmentation of the functional network into a set of strongly intra- but weakly interconnected subnetworks, which is followed by reconfiguration into a smaller set of weakly intraconnected subnetworks towards seizure end 
(Schindler et al., 2008; Kramer et al., 2008, 2010; Jiruska et al., 2013). Moreover, the topology of ictal networks was found to be more ordered (lattice like), compared to the preand postictal networks. This view is based mainly on a characteristic rise and fall profile of the epoch courses of the number of connected network components, normalized clustering coefficient and the normalized characteristic path length (Schindler et al., 2008; Kramer et al., 2010; van Diessen et al., 2013). Clearly, the hilly epoch courses of the modularity and normalized clustering coefficient of our predicted networks also exhibit a rise and fall profile and thus are consistent with the fragmentation\reconfiguration idea (see figure 8, high $T \geq p_{75}$ ). A further measure whose epoch course was reproduced in this study is the edge density. In (Kramer et al., 2010) epoch course of the edge density was shown to become slightly depressed after ictal onset and remaining so for the most time, until a dramatic rise setting in towards seizure end. Again, this is exactly the profile displayed by our predicted networks in figure 8 (for high $T$ ). Comparison of our epoch courses with those in (Kramer et al., 2010) is valid, although in the latter case the edge density was computed for the full network, whereas we considered the mean edge density across network components (this was necessary since the edge density of our full networks is epoch independent and given by the threshold percentile percent). Validity is given, because on average the largest component of our networks comprised $75-82 \%$ of the nodes, while it displayed the same edge density epoch profile as in figure 8 for $p_{75}, p_{90}$ (not shown). Therefore, the by far largest part of the predicted networks showed an edge density profile identical to (Kramer et al., 2010).

When all examined network measures are taken into account, setting $T=p_{90}$ yields the best overall, qualitative match between our results and those reported in (Kramer et al., 2010), while at the same time excellent fits of the CL functional networks to the ones computed from the raw data are produced.

One interpretation of these findings is that during seizure progression epileptic brain areas fragment into assemblies("islands") of hypersynchronized neurons that are relatively independent (desynchronized) from neurons in other such assemblies. Along different lines of evidence, moreover, eigenvector analysis has indicated that towards seizure end such an initial desynchronization is followed by a reconfiguring hypersynchronization phase, which may thus even play a beneficial role as a mechanism for seizure termination (Schindler et al., 2007). These findings constitute a departure from the traditional view, which considers seizures as events of temporally constant, global hypersynchrony (Schindler et al., 2007). Also, our result regarding the epoch course of the Shannon entropy lends further support against the hypothesis of constant, global hypersynchrony during seizures, since synchronous processes are associated with low entropy which, in contrast, we found to be largest at ictal mid-time. In the light of the fragmentation\reconfiguration hypothesis, this may be interpreted as the global fragmentation process (that tends to desynchronize neuronal assemblies) being the stronger one, compared to the local synchronization processes that occur simultaneously within any such assembly. Reconfiguring hypersynchronization that putatively follows such an initial phase of network fragmentation is possibly reflected by edge-density and mean edge-weight profiles that both become strongest towards seizure end, together with a modularity that by then has fallen even below preictal levels (figure $8, T=p_{90}$ ). This 
way, a defragmented, monolithic network with strong weights emerges that is indicative of a hypersynchronization process.

On the other hand, the epoch profile of the normalized characteristic path length in (Kramer et al., 2010) was slightly different from the rise and fall profile shown in this study (for $T=p_{90}$ ), most notably because the formers maximum occurred in the postictal epoch. (Schindler et al., 2008) in contrast also reported a rise and fall profile that is consistent with ours. The reason for these inconsistencies remains unknown. However one has to notice that the functional networks in the reported literature were constructed in different ways; In (Schindler et al., 2008; Kramer et al., 2010) the networks were unweighted and binary (an edge was either present or not present but had no edge weight), unlike the weighted networks we have considered in this study. Also, the thresholding procedure in (Kramer et al., 2010) was based on correlation significance, while in (Schindler et al., 2008) a correlation threshold was lowered as much as possible, such that only a single network component was maintained. These threshold definitions are different from our percentile based one and, together with the presence of binary edges, may explain the small differences between results (Stam et al., 2014). However, we could not rely on the reported threshold definitions, because (a) our functional networks were inferred from a predictive model and thus no information regarding correlation significance was present and (b) comparisons with respect to fragmentation would not have been meaningful had we maintained a single network component only. On the other hand, if the hypothesized fragmentation\reconfiguration process is a hallmark of epileptic seizures, one expects related analysis procedures to reach similar conclusions and in this study we have found several lines of evidence supporting this scenario. We have also laid out explicitly (figures 8\&9) that our results inferred from the CL-model cannot be solely explained by the correlations that are trivially matched by the CL-algorithm, rather they are emergent properties of the resulting CL-tree.

The significance of the latter as a predictive model lies in its simplicity, which allows for efficient and exact Bayesian inference, in contrast to e.g. graphical models (GMs) containing cycles (Löliger, 2004). There have been a few clinically oriented studies that applied GMs to general EEG and magnetoencephalography time-series (Shenoy \& Rao, 2005; Nagarajan et al., 2006; Iyengar et al., 2010; Dauwels et al., 2012), but there have been surprisingly few such studies in the context of epilepsy research (Dauwels et al., 2011; Wulsin et al., 2013). In (Dauwels et al., 2011) a GM-based classifier was trained to segregate EEG signals recorded from the seizure-onset-zone (SOZ) from those recorded outside the SOZ. Importantly, this approach implicitly assumes the existence of a single SOZ, moreover, one that is unequivocally definable by human experts. Yet it is often not possible to clearly define a SOZ (Bartolomei et al., 2013). Furthermore, the approaches of (Dauwels et al., 2012, 2011) rely on the totality of EEG time-series for GM learning and do not consider the latters temporal evolution. However, a spatio-temporal model of EEG activity may be crucial for understanding seizure dynamics (Direito et al., 2012).

The CL model in contrast can be naturally extended to become the "Mixture of trees" model (Meila \& Jordan, 2001), which enables a straightforward generalization towards the GMbased modeling of dynamic quantities, an approach that has already been applied success- 
fully in the context of non-EEG time series (Kirshner et al., 2004). The basic idea behind this generalization is to condense the temporal evolution of all dynamic variables (iEEG channels) into a single unobserved ("hidden") variable, which evolves according to a Hidden Markov Model (HMM) (Rabiner \& Juang, 1986; Barber \& Cemgil, 2010). Given a collection of CL-trees, the hidden variable can be interpreted as a tree-selector variable, whose posterior probability distribution determines the degree of responsibility of each tree for producing a data point. In the context of iEEG time-series, different trees may thus absorb the spatial dependencies of different time windows (as has been done in this study), whereas the hidden variable serves the purpose of modeling the temporal dependencies of the series, by establishing relationships between neighboring time windows.

This approach is particularly promising in the context of epileptic seizures, since the latter are characterized by rapid transitions from non-seizure to seizure brain states, an aspect that can be incorporated by HMMs through a fast redistribution of the hidden variables posterior probability mass (i.e. from CL-trees representing non-seizure to CL-trees representing seizure brain states). Such a combined setup, consisting of a HMM in conjunction with a set of CL-trees, may be suitable also for the modeling of brain diseases other than epilepsy. However, we believe the existence of global changes in brain state (like seizures) to be a necessary precondition for the applicability of such a model. As mentioned before, this is because a HMM condenses all temporal evolution into a single hidden variable and is thus conceptually unable to model state changes that occur asynchronously in different brain regions. There have been a limited number of studies only that applied HMMs to general (Lederman \& Tabrikian, 2012) and epileptiform EEG time-series (Santaniello et al., 2011; Direito et al., 2012; Wulsin et al., 2013). Except for the very recent study by (Wulsin et al., 2013), the generative and predictive power of these approaches has been confined mainly to the temporal, rather than spatio-temporal domain and hence none of these studies yielded a fully generative or predictive model for the dynamics of high-dimensional iEEG time-series. Even the model presented by (Direito et al., 2012) is not fully generative or predictive in the spatial domain, since it does not allow to draw any inferences about only a subset of the EEG electrodes, given that the other electrodes are clamped to specific states. In the context of epilepsy therapies however, such a setup is highly desirable, since it could be a starting point for the development of brain stimulation protocols, by allowing to predict $\backslash$ test their impact on iEEG dynamics. Therefore, the development of a CL-based HMM is the most immediate amongst our future working steps.

Furthermore, we have plans to apply the resulting model to iEEG data restricted by different frequency bands. Indeed, it is possible that the results presented in this paper hinge at the presence of some particular frequency bands, such that relevant correlations are caused mainly by activity at these frequencies. In (Weiss et al., 2013) for example, crossfrequency coupling between low-frequencies $(\leq 25 \mathrm{~Hz})$ and the high-gamma range $(>80 \mathrm{~Hz})$ is shown for the "ictal core" region -the region that is directly affected by an ictal wavefront and where neurons show characteristic bursts- but not for its periphery, called the "penumbra". In principle, the CL-algorithm may also detect such rather subtle dependencies, thereby transferring predictive modeling even further to the spatio-temporal and frequency domain. 


\section{Acknowledgements}

This work was supported by the Swiss National Science Foundation (SNF) (Project No: SNF 32003B_155950). The authors declare no conflict of interest. Neither the authors current employer (Inselspital Bern/Switzerland) nor any of their previous employers influenced this studys design, data collection, analysis and interpretation, the writing of the manuscript and the decision to submit the article for publication.

\section{References}

Aji, M., \& McEliece, R. (2000, March). The Generalized Distributive Law. IEEE Transactions on Information Theory, 46(2), 325-343.

Barber, D., \& Cemgil, A. (2010, November). Graphical Models for Time-Series. IEEE Signal Processing Magazine, 27(6), 18-28.

Bartolomei, F., Guye, M., \& Wendling, F. (2013). Abnormal binding and disruption in large scale networks involved in human partial seizures. Nonlinear biomedical physics.

Bishop, C. (2006). Pattern Recognition and Machine Learning. Springer Science+Business Media.

Bland, M. (2013, October). Do Baseline P-Values Follow a Uniform Distribution in Randomised Trials? PLoS one, 8(10), 1-5.

Bozorgi, A., \& Lhatoo, S. D. (2013). Seizures, Cerebral Shutdown, and SUDEP. Epilepsy Currents / American Epilepsy Society, 13(5), 236-40. doi: 10.5698/1535-7597-13.5.236

Bullmore, E., \& Sporns, O. (2009). Complex brain networks: graph theoretical analysis of structural and functional systems. Nature Reviews Neuroscience, 10(3), 186-98. doi: $10.1038 / \operatorname{nrn} 2575$

Cascino, G. D. (2008). When drugs and surgery don't work. Epilepsia, 49 Suppl 9, 79-84. doi: $10.1111 /$ j.1528-1167.2008.01930.x

Chow, C., \& Liu, C. (1968). Approximating Discrete Probability Distributions with Dependence Trees. IEEE Transactions on Information Theory, IT-14(3), 462-467.

Dauwels, J., Eskandar, E., Cole, A., Hoch, D., Zepeda, R., \& Cash, S. (2011). Graphical models for localization of the seizure focus from interictal intracranial EEG. In IEEE International Conference on Acoustics Speech and Signal Processing (p. 745-748). Prague.

Dauwels, J., Yu, H., Wang, X., Vialette, F., Latchoumane, C.-F., Jeong, J., \& Cichocki, A. (2012). Inferring Brain Networks through Graphical Models with Hidden Variables. In (Machine Learning and Interpretation in Neuroimaging, Lecture Notes in Computer Science ed., Vol. 7263, p. 194-201). 
Direito, B., Teixera, C., Ribeiro, B., Castelo-Branco, M., \& Sales, F. (2012). Modeling epileptic brain states using EEG spectral analysis and topographic mapping. Journal of Neuroscience Methods, 210, 220-229.

Engel, J., Jr, Thompson, P. M., Stern, J. M., Staba, R. J., Bragin, A., \& Mody, I. (2013). Connectomics and epilepsy. Current Opinion in Neurology. doi: 10.1097/ WCO.0b013e32835ee5b8

Fisher, R. S., van Emde Boas, W., Blume, W., Elger, C., Genton, P., Lee, P., \& Engel, J., J. (2005). Epileptic seizures and epilepsy: definitions proposed by the international league against epilepsy (ilae) and the international bureau for epilepsy (ibe). Epilepsia, 46(4), 470-2. doi: $10.1111 / \mathrm{j} .0013-9580.2005 .66104 . \mathrm{x}$

Iyengar, S., Dauwels, J., Varshney, P., \& Cichocki, A. (2010). Quantifying EEG Synchrony Using Copulas. In IEEE International Conference on Acoustics Speech and Signal Processing (p. 505-508). Dallas, TX.

Jiruska, P., de Curtis, M., Jeffreys, J., Schevon, S., C.A. Schiff, \& Schindler, K. (2013). Synchronization and desynchronization in epilepsy: controversies and hypotheses. Journal of Physiology, 591.4, 787-797.

Kirshner, S., Smyth, P., \& Robertson, A. (2004). Conditional Chow-Liu Tree Structures for Modeling Discrete-Valued Vector Time Series. In Proceedings of the Twentieth Conference on Uncertainty in Artificial Intelligence (UAI).

Koller, D., \& Friedman, N. (2009). Probabilistic Graphical Models (T. Dietterich, Ed.). Cambridge Massachusetts: MIT Press.

Kramer, M. A., Eden, U. T., Kolaczyk, E. D., Zepeda, R., Eskandar, E. N., \& Cash, S. S. (2010). Coalescence and fragmentation of cortical networks during focal seizures. Journal of Neuroscience, 30(30), 10076-10085. doi: 10.1523/jneurosci.6309-09.2010

Kramer, M. A., Kolaczyk, E. D., \& Kirsch, H. E. (2008). Emergent network topology at seizure onset in humans. Epilepsy Research, 79(2-3), 173-86. doi: 10.1016/j.eplepsyres .2008 .02 .002

Kruskal, J. (1956). On the shortest spanning subtree of a graph and the traveling salesman problem. Proc. Am. Math. Soc., 7(1), 48-50.

Lederman, D., \& Tabrikian, J. (2012). Classification of multichannel eeg patterns using parallel hidden markov models. Medical \&3 Biological Engineering $\& 3$ Computing, 50(4), 319-328.

Löliger, H. (2004, January). An Introduction to Factor Graphs. IEEE Signal Proc. Mag., 21, 28-41. 
Meila, M., \& Jordan, M. (2001). Learning With Mixtures of Trees. The Journal of Machine Learning Research, 1, 1-48.

Meyer, A., Dua, T., Ma, J., Saxena, S., \& Birbeck, G. (2010). Global disparities in the epilepsy treatment gap: a systematic review. Bulletin of the World Health Organization, $88,260-266$.

Mormann, F., Andrzejak, R. G., Elger, C. E., \& Lehnertz, K. (2007). Seizure prediction: the long and winding road. Brain : a journal of neurology, 130(Pt 2), 314-33. doi: $10.1093 /$ brain/awl241

Murphy, K. (2012). Machine Learning: a Probabilistic Perspective. MIT Press.

Nagarajan, S., Attias, H., Hild, K., \& Sekihara, K. (2006). A Graphical Model for Estimating Stimulus-Evoked Brain Responses in Noisy MEG data with Large Background Brain Activity. NeuroImage, 30(2), 400-416.

Newman, M. E. J. (2006, Sep). Finding community structure in networks using the eigenvectors of matrices. Phys. Rev. E, 74, 036104. doi: 10.1103/PhysRevE.74.036104

Onnela, J., Saramaki, J., Kertesz, J., \& Kaski, K. (2005). Intensity and coherence of motifs in weighted complex networks. Physiol. Rev. E, Stat. Nonlinear Soft Matter Phys., 71.

Ponten, S. C., Bartolomei, F., \& Stam, C. J. (2007). Small-world networks and epilepsy: graph theoretical analysis of intracerebrally recorded mesial temporal lobe seizures. Clinical Neurophysiology : Official Journal of the International Federation of Clinical Neurophysiology, 118(4), 918-27. doi: 10.1016/j.clinph.2006.12.002

Prim, R. (1957). Shortest connection networks and some generalizations. Bell Syst. Tech. J., $36,1389-1401$.

Rabiner, L., \& Juang, B. (1986, January). An Introduction to Hidden markov Models. IEEE ASSP Magazine, 3, 4-16.

Richardson, M. P. (2012). Large scale brain models of epilepsy: dynamics meets connectomics. Journal of neurology, neurosurgery, and psychiatry. doi: 10.1136/jnnp-2011 $-301944$

Rubinov, M., Kötter, R., Hagmann, P., \& Sporns, O. (2009, July). Brain connectivity toolbox: a collection of complex network measurements and brain connectivity datasets. NeuroImage, 47, S169. doi: 10.1016/s1053-8119(09)71822-1

Rubinov, M., \& Sporns, O. (2010). Complex network measures of brain connectivity: Uses and interpretations. NeuroImage, 52(3), 1059-1069. doi: 10.1016/j.neuroimage.2009.10 .003 
Santaniello, S., Sherman, D., Mirski, M., Thakor, N., \& Sarma, S. (2011). A Bayesian framework for analyzing iEEG data from a rat model of epilepsy. In IEEE Engineering in Medicine and Biology Society Conference (p. 1435-1438).

Schindler, K., Bialonski, S., Horstmann, M.-T., Elger, C., \& Lehnertz, K. (2008). Evolving functional network properties and synchronizability during human epileptic seizures. Chaos, 18(3), 033119.

Schindler, K., Leung, H., Elger, C. E., \& Lehnertz, K. (2007). Assessing seizure dynamics by analysing the correlation structure of multichannel intracranial eeg. Brain, 130 (Pt 1), 65-77. doi: awl304[pii]10.1093/brain/awl304

Schreiber, T., \& Schmitz, A. (2000). Surrogate Time Series. Physica D, 142, 346-382.

Shenoy, P., \& Rao, R. P. (2005). Dynamic bayesian networks for brain-computer interfaces. In L. Saul, Y. Weiss, \& L. Bottou (Eds.), Advances in neural information processing systems 17 (pp. 1265-1272). MIT Press.

Stam, C., Tewarie, P., van Dellen, E., van Straaten, E. C., Hillebrand, A., \& van Mieghem, P. (2014). The trees and the forest: Characterization of complex brain networks with minimum spanning trees. International Journal of Psychophysiology, 92, 129-138.

Stam, C., \& van Straaten, E. (2012). The organization of physiological brain networks. Clinical neurophysiology : official journal of the International Federation of Clinical Neurophysiology, 123(6), 1067-87. doi: 10.1016/j.clinph.2012.01.011

van Diessen, E., Diederen, S., Braun, K., Jansen, F., \& Stam, C. (2013). Functional and structural brain networks in epilepsy: What have we learned? Epilepsia, 54(11), 18551865.

van Mierlo, P., Carrette, E., Hallez, H., Vonck, K., van Roost, D., Boon, P., \& Staelens, S. (2011). Accurate epileptogenic focus localization through time-variant functional connectivity analysis of intracranial electroencephalographic signals. NeuroImage, 56, 1122-1133.

Varotto, G., Tassi, L., Franceschetti, S., Spreafico, R., \& Panzica, F. (2012). Epileptogenic networks of type II focal cortical dysplasia: A stereo-EEG study. NeuroImage, 61, 591598.

Weiss, S., Banks, G., Jr, G. M., Goodman, R., Emerson, R., Trevelyan, A., \& Schevon, C. (2013, December). Ictal high frequency oscillations distinguish two types of seizure territories in humans. Brain, 136, 1-15.

Wilke, C., Worrell, G., \& He, B. (2011). Graph analysis of epileptogenic networks in human partial epilepsy. Epilepsia, 52(1), 84-93. doi: 10.1111/j.1528-1167.2010.02785.x 
Wulsin, D., Fox, E., \& Litt, B. (2013). Parsing Epileptic Events Using a Markov Switching Process for Correlated Time Series (Vol. 28) (No. 30). Proceedings of the 30th International Conference on Machine Learning, Atlanta, Georgia, USA.

Zubler, F., Gast, H., Abela, E., Rummel, C., Hauf, M., Wiest, R., .. Schindler, K. (2014, May). Detecting Functional Hubs of Ictogenic Networks. Brain Topography. 